Research Article

\title{
Supercritical Neimark-Sacker Bifurcation and Hybrid Control in a Discrete-Time Glycolytic Oscillator Model
}

\author{
A. Q. Khan $\mathbb{D D}^{1}{ }^{1}$ E. Abdullah, ${ }^{1}$ and Tarek F. Ibrahim ${ }^{2,3}$ \\ ${ }^{1}$ Department of Mathematics, University of Azad Jammu \& Kashmir, Muzaffarabad 13100, Pakistan \\ ${ }^{2}$ Department of Mathematics, Faculty of Sciences and Arts in Mahayel Aseer, King Khalid University, Abha, Saudi Arabia \\ ${ }^{3}$ Department of Mathematics, Faculty of Sciences, Mansoura University, Mansoura 35516, Egypt
}

Correspondence should be addressed to A. Q. Khan; abdulqadeerkhan1@gmail.com

Received 18 September 2019; Accepted 30 December 2019; Published 22 January 2020

Academic Editor: Massimiliano Ferrara

Copyright ( 92020 A. Q. Khan et al. This is an open access article distributed under the Creative Commons Attribution License, which permits unrestricted use, distribution, and reproduction in any medium, provided the original work is properly cited.

We study the local dynamical properties, Neimark-Sacker bifurcation, and hybrid control in a glycolytic oscillator model in the interior of $\mathbb{R}_{+}^{2}$. It is proved that, for all parametric values, $P_{x y}^{+}\left(\alpha /\left(\beta+\alpha^{2}\right), \alpha\right)$ is the unique positive equilibrium point of the glycolytic oscillator model. Further local dynamical properties along with different topological classifications about the equilibrium $P_{x y}^{+}\left(\alpha /\left(\beta+\alpha^{2}\right), \alpha\right)$ have been investigated by employing the method of linearization. Existence of prime period and periodic points of the model under consideration are also investigated. It is proved that, about the fixed point $P_{x y}^{+}\left(\alpha /\left(\beta+\alpha^{2}\right), \alpha\right)$, the discrete-time glycolytic oscillator model undergoes no bifurcation, except Neimark-Sacker bifurcation. A further hybrid control strategy is applied to control Neimark-Sacker bifurcation in the discrete-time model. Finally, theoretical results are verified numerically.

\section{Introduction}

In glycolysis, glucose decomposes in the presence of various enzymes including ten steps in which five are termed the preparatory phase or phosphorylation, while the remaining steps are called the pay-off phase. Phosphofructokinase is one of the enzymes which is responsible for the occurrence of glycolytic oscillation [1-4]. This step is considered the control unit of glycolysis due to the presence of enzyme phosphofructokinase. A biochemical reaction that occurs in metabolic systems has the following sequence of steps [5]:

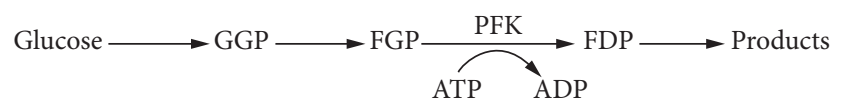

where the quantities involved in the above biochemical reaction are depicted in Table 1.

Generally, it is assumed that the enzyme PFK consists of two steps as given below. Among these, in one step, adenosine diphosphate simulates the allosteric regulatory enzyme and yields a more active form. Therefore, the product formed during the reaction mediated by phosphofructokinase increases the rate of reaction. The systematic description of the kinetics is as follows:

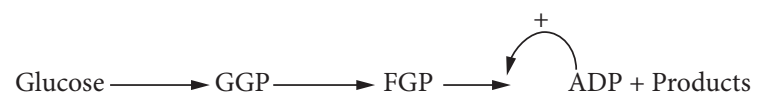

and we have

$$
\begin{aligned}
& \frac{\mathrm{d} x}{\mathrm{~d} t}=\alpha-\beta x-x y^{2}, \\
& \frac{\mathrm{d} y}{\mathrm{~d} t}=\beta x+x y^{2}-y,
\end{aligned}
$$

where $x_{n}$ and $y_{n}$ denote FGP and ADP, respectively, and $\alpha$ and $\beta$ are positive constants. Using the nonstandard finite difference scheme, the discrete analogue of the glycolytic oscillator model, which is depicted in (3), is 
TABLE 1: Quantities along their chemical interpretation.

\begin{tabular}{lc}
\hline Quantities & Corresponding chemical interpretation \\
\hline GGP & Glucose-6-phosphate \\
FGP & Fructose-6-phosphate \\
FDP & Fructose-1,6-diphosphate \\
PFK & Phosphofructokinase \\
ATP & Adenosine triphosphate \\
ADP & Adenosine diphosphate \\
\hline
\end{tabular}

$$
\begin{aligned}
& x_{n+1}=\frac{\alpha h+x_{n}}{1+h \beta+h y_{n}^{2}}, \\
& y_{n+1}=\frac{y_{n}+h \beta x_{n}+h x_{n} y_{n}^{2}}{1+h} .
\end{aligned}
$$

Here, our purpose is to explore the local dynamics, N-S bifurcation, and hybrid control in a glycolytic oscillator model (4) in the interior of $\mathbb{R}_{+}^{2}$. Specifically, our key findings are as follows:

(1) About the unique +ve fixed point $P_{x y}^{+}\left(\alpha /\left(\beta+\alpha^{2}\right), \alpha\right)$, we explored the dynamical properties along with different topological classifications.

(2) We explored the existence of bifurcation about $P_{x y}^{+}\left(\alpha /\left(\beta+\alpha^{2}\right), \alpha\right)$.

(3) Prime period and periodic points of the glycolytic oscillator model (4) are investigated.

(4) To prove, the glycolytic oscillator model (4) undergoes an N-S bifurcation when parameters vary in a small neighborhood of $P_{x y}^{+}\left(\alpha /\left(\beta+\alpha^{2}\right), \alpha\right)$, and no other bifurcation occurs except the N-S bifurcation.

(5) The N-S bifurcation is controlled by the hybrid control strategy.

(6) Theoretical results are verified numerically.

The flow pattern of the remaining of this paper is as follows: Section 2 is about the existence of the unique +ve equilibrium point and corresponding linearized form of the glycolytic oscillator model (4). In Section 3, we study the local dynamical properties with topological classification about $P_{x y}^{+}\left(\alpha /\left(\beta+\alpha^{2}\right), \alpha\right)$ of the glycolytic oscillator model (4), whereas existence of prime period and periodic points of model (4) is explored in Section 4. The comprehensive N-S bifurcation analysis about $P_{x y}^{+}\left(\alpha /\left(\beta+\alpha^{2}\right), \alpha\right)$ is given in Section 5. In Section 6, simulations are presented to verify theoretical results. In Section 7, a hybrid control strategy is applied to control N-S bifurcation. Concluding remarks are given in Section 8.

\section{Existence of the Unique +ve Equilibrium Point and Corresponding Linearized Form of the Glycolytic Oscillator Model (4)}

The existence of the +ve fixed point in the interior of $\mathbb{R}_{+}^{2}$ and corresponding linearized form of the glycolytic oscillator model (4) are explored in this section. More specifically, the existence result about the equilibrium point can be summarized as follows.

Lemma 1. $\forall$ parametric values $\alpha$ and $\beta$, the discrete-time glycolytic oscillator model (4) has a unique +ve equilibrium point $P_{x y}^{+}\left(\alpha /\left(\beta+\alpha^{2}\right), \alpha\right)$.

Now, the linearized form of the glycolytic oscillator model (4) about $P_{x y}(x, y)$ under the map $\left(\Psi_{1}, \Psi_{2}\right) \longrightarrow$ $\left(x_{n+1}, y_{n+1}\right)$ becomes

$$
\Lambda_{n+1}=\left.J\right|_{P_{x y}(x, y)} \Lambda_{n}
$$

where

$$
\begin{aligned}
\left.J\right|_{P_{x y}(x, y)} & =\left(\begin{array}{cc}
\frac{1}{1+h\left(\beta+y^{2}\right)} & \frac{-2 y h(x+\alpha h)}{\left(1+h\left(\beta+y^{2}\right)\right)^{2}} \\
\frac{h\left(\beta+y^{2}\right)}{1+h} & \frac{1+2 h x y}{1+h}
\end{array}\right), \\
\Psi_{1} & =\frac{\alpha h+x_{n}}{1+h \beta+h y_{n}^{2}}, \\
\Psi_{2} & =\frac{y_{n}+h \beta x_{n}+h x_{n} y_{n}^{2}}{1+h} .
\end{aligned}
$$

\section{Local Dynamical Properties of Model \\ (4) about $P_{x y}^{+}\left(\alpha /\left(\beta+\alpha^{2}\right), \alpha\right)$}

The local dynamics about $P_{x y}^{+}\left(\alpha /\left(\beta+\alpha^{2}\right), \alpha\right)$ of the glycolytic oscillator model (4) is explored in this section. $\left.J\right|_{P_{x y}^{+}\left(\alpha /\left(\beta+\alpha^{2}\right), \alpha\right)}$ about $P_{x y}^{+}\left(\alpha /\left(\beta+\alpha^{2}\right), \alpha\right)$ is

$$
\left.J\right|_{P_{x y}^{+}\left(\alpha /\left(\beta+\alpha^{2}\right), \alpha\right)}=\left(\begin{array}{cc}
\frac{1}{1+h\left(\beta+\alpha^{2}\right)}-\frac{2 \alpha^{2} h}{\left(\alpha^{2}+\beta\right)\left(1+h\left(\beta+\alpha^{2}\right)\right)} \\
\frac{h\left(\beta+\alpha^{2}\right)}{1+h} & \frac{\alpha^{2}+\beta+2 h \alpha^{2}}{\left(\beta+\alpha^{2}\right)(1+h)}
\end{array}\right) .
$$

The auxiliary equation of $\left.J\right|_{P_{x y}^{+}\left(\alpha /\left(\beta+\alpha^{2}\right), \alpha\right)}$ about $P_{x y}^{+}\left(\alpha /\left(\beta+\alpha^{2}\right), \alpha\right)$ is

$$
\lambda^{2}-p\left(\frac{\alpha}{\beta+\alpha^{2}}, \alpha\right) \lambda+q\left(\frac{\alpha}{\beta+\alpha^{2}}, \alpha\right)=0,
$$

where

$$
\begin{aligned}
& p\left(\frac{\alpha}{\beta+\alpha^{2}}, \alpha\right)=\frac{(1+h)\left(\beta+\alpha^{2}\right)+\left(\alpha^{2}+\beta+2 h \alpha^{2}\right)\left(1+h\left(\alpha^{2}+\beta\right)\right)}{\left(1+h\left(\alpha^{2}+\beta\right)\right)(1+h)\left(\beta+\alpha^{2}\right)}, \\
& q\left(\frac{\alpha}{\beta+\alpha^{2}}, \alpha\right)=\frac{\alpha^{2}+\beta+2 h \alpha^{2}+2 h^{2} \alpha^{2}\left(\alpha^{2}+\beta\right)}{(1+h)\left(\alpha^{2}+\beta\right)\left(1+h\left(\beta+\alpha^{2}\right)\right)} .
\end{aligned}
$$$$
\text { And eigenvalues of }\left.J\right|_{P_{x y}^{+}\left(\alpha /\left(\beta+\alpha^{2}\right), \alpha\right)} \text { about } P_{x y}^{+}\left(\alpha /\left(\beta+\alpha^{2}\right), \alpha\right)
$$
are

$$
\lambda_{1,2}=\frac{p\left(\alpha /\left(\beta+\alpha^{2}\right), \alpha\right) \pm \sqrt{\Delta}}{2},
$$


where

$$
\begin{aligned}
\Delta= & \left(p\left(\frac{\alpha}{\beta+\alpha^{2}}, \alpha\right)\right)^{2}-4 q\left(\frac{\alpha}{\beta+\alpha^{2}}, \alpha\right) \\
= & \left(\frac{(1+h)\left(\beta+\alpha^{2}\right)+\left(\alpha^{2}+\beta+2 h \alpha^{2}\right)\left(1+h\left(\alpha^{2}+\beta\right)\right)}{\left(1+h\left(\alpha^{2}+\beta\right)\right)(1+h)\left(\beta+\alpha^{2}\right)}\right)^{2} \\
& -4\left(\frac{\alpha^{2}+\beta+2 h \alpha^{2}+2 h^{2} \alpha^{2}\left(\alpha^{2}+\beta\right)}{(1+h)\left(\alpha^{2}+\beta\right)\left(1+h\left(\beta+\alpha^{2}\right)\right)}\right) .
\end{aligned}
$$

Hereafter in the following, we will explore the necessary and sufficient condition under which $P_{x y}^{+}\left(\alpha /\left(\beta+\alpha^{2}\right), \alpha\right)$ of the glycolytic oscillator model (4) is a sink, a repeller, a saddle, and nonhyperbolic.

Lemma 2. For $P_{x y}^{+}\left(\alpha /\left(\beta+\alpha^{2}\right), \alpha\right)$ of the glycolytic oscillator model (4), the following holds:

(i) $P_{x y}^{+}\left(\alpha /\left(\beta+\alpha^{2}\right), \alpha\right)$ is a sink iff

$$
\begin{aligned}
& \left|\frac{(1+h)\left(\beta+\alpha^{2}\right)+\left(\alpha^{2}+\beta+2 h \alpha^{2}\right)\left(1+h\left(\alpha^{2}+\beta\right)\right)}{\left(1+h\left(\alpha^{2}+\beta\right)\right)(1+h)\left(\beta+\alpha^{2}\right)}\right| \\
& <1-\frac{\alpha^{2}+\beta+2 h \alpha^{2}+2 h^{2} \alpha^{2}\left(\alpha^{2}+\beta\right)}{(1+h)\left(\alpha^{2}+\beta\right)\left(1+h\left(\beta+\alpha^{2}\right)\right)}<2 .
\end{aligned}
$$

(ii) $P_{x y}^{+}\left(\alpha /\left(\beta+\alpha^{2}\right), \alpha\right)$ is a repeller iff

$$
\begin{aligned}
& \left|\frac{\alpha^{2}+\beta+2 h \alpha^{2}+2 h^{2} \alpha^{2}\left(\alpha^{2}+\beta\right)}{(1+h)\left(\alpha^{2}+\beta\right)\left(1+h\left(\beta+\alpha^{2}\right)\right)}\right|>1, \\
& \left|\frac{(1+h)\left(\beta+\alpha^{2}\right)+\left(\alpha^{2}+\beta+2 h \alpha^{2}\right)\left(1+h\left(\alpha^{2}+\beta\right)\right)}{\left(1+h\left(\alpha^{2}+\beta\right)\right)(1+h)\left(\beta+\alpha^{2}\right)}\right| \\
& \quad<\left|1-\frac{\alpha^{2}+\beta+2 h \alpha^{2}+2 h^{2} \alpha^{2}\left(\alpha^{2}+\beta\right)}{(1+h)\left(\alpha^{2}+\beta\right)\left(1+h\left(\beta+\alpha^{2}\right)\right)}\right| .
\end{aligned}
$$

(iii) $P_{x y}^{+}\left(\alpha /\left(\beta+\alpha^{2}\right), \alpha\right)$ is a saddle iff

$$
\begin{aligned}
& \left(\frac{(1+h)\left(\beta+\alpha^{2}\right)+\left(\alpha^{2}+\beta+2 h \alpha^{2}\right)\left(1+h\left(\alpha^{2}+\beta\right)\right)}{\left(1+h\left(\alpha^{2}+\beta\right)\right)(1+h)\left(\beta+\alpha^{2}\right)}\right)^{2} \\
& +4\left(1-\frac{\alpha^{2}+\beta+2 h \alpha^{2}+2 h^{2} \alpha^{2}\left(\alpha^{2}+\beta\right)}{(1+h)\left(\alpha^{2}+\beta\right)\left(1+h\left(\beta+\alpha^{2}\right)\right)}\right)>0, \\
& \left|\frac{(1+h)\left(\beta+\alpha^{2}\right)+\left(\alpha^{2}+\beta+2 h \alpha^{2}\right)\left(1+h\left(\alpha^{2}+\beta\right)\right)}{\left(1+h\left(\alpha^{2}+\beta\right)\right)(1+h)\left(\beta+\alpha^{2}\right)}\right| \\
& >\left|1-\frac{\alpha^{2}+\beta+2 h \alpha^{2}+2 h^{2} \alpha^{2}\left(\alpha^{2}+\beta\right)}{(1+h)\left(\alpha^{2}+\beta\right)\left(1+h\left(\beta+\alpha^{2}\right)\right)}\right| .
\end{aligned}
$$

(iv) $P_{x y}^{+}\left(\alpha /\left(\beta+\alpha^{2}\right), \alpha\right)$ is nonhyperbolic iff

$$
\begin{aligned}
& \left|\frac{(1+h)\left(\beta+\alpha^{2}\right)+\left(\alpha^{2}+\beta+2 h \alpha^{2}\right)\left(1+h\left(\alpha^{2}+\beta\right)\right)}{\left(1+h\left(\alpha^{2}+\beta\right)\right)(1+h)\left(\beta+\alpha^{2}\right)}\right| \\
& \quad=\left|1-\frac{\alpha^{2}+\beta+2 h \alpha^{2}+2 h^{2} \alpha^{2}\left(\alpha^{2}+\beta\right)}{(1+h)\left(\alpha^{2}+\beta\right)\left(1+h\left(\beta+\alpha^{2}\right)\right)}\right| .
\end{aligned}
$$

Proof. Follows from Theorem 1.1.1 of [6].

Hereafter in the next lemma, we will explore local dynamics along with topological classification about $P_{x y}^{+}(\alpha /$ $\left.\left(\beta+\alpha^{2}\right), \alpha\right)$ if $\Delta=\left(\left((1+h)\left(\beta+\alpha^{2}\right)+\left(\alpha^{2}+\beta+2 h \alpha^{2}\right)(1+\right.\right.$ $\left.\left.h\left(\alpha^{2}+\beta\right)\right)\right) /\left(\left(1+h\left(\alpha^{2}+\beta\right)(1+h)\left(\beta+\alpha^{2}\right)\right)\right)^{2}-4\left(\left(\alpha^{2}+\beta+\right.\right.$ $\left.\left.2 h \alpha^{2}+2 h^{2} \alpha^{2}\left(\alpha^{2}+\beta\right)\right) /\left((1+h)\left(\alpha^{2}+\beta\right)\left(1+h\left(\beta+\alpha^{2}\right)\right)\right)\right)<0$, of the glycolytic oscillator model (4).

Lemma 3. If $\Delta=\left(\left((1+h)\left(\beta+\alpha^{2}\right)+\left(\alpha^{2}+\beta+2 h \alpha^{2}\right)(1+\right.\right.$ $\left.\left.h\left(\alpha^{2}+\beta\right)\right)\right) /\left(\left(1+h\left(\alpha^{2}+\beta\right)(1+h)\left(\beta+\alpha^{2}\right)\right)\right)^{2}-4\left(\left(\alpha^{2}+\beta+\right.\right.$ $\left.\left.2 h \alpha^{2}+2 h^{2} \alpha^{2}\left(\alpha^{2}+\beta\right)\right) /\left((1+h)\left(\alpha^{2}+\beta\right)\left(1+h\left(\beta+\alpha^{2}\right)\right)\right)\right)<$ 0 , then for $P_{x y}^{+}\left(\alpha /\left(\beta+\alpha^{2}\right), \alpha\right)$, the following holds:

(i) $P_{x y}^{+}\left(\alpha /\left(\beta+\alpha^{2}\right), \alpha\right)$ is a locally asymptotically stable focus if

$$
\frac{\alpha^{2}+\beta+2 h \alpha^{2}+2 h^{2} \alpha^{2}\left(\alpha^{2}+\beta\right)}{(1+h)\left(\alpha^{2}+\beta\right)\left(1+h\left(\beta+\alpha^{2}\right)\right)}<1 .
$$

(ii) $P_{x y}^{+}\left(\alpha /\left(\beta+\alpha^{2}\right), \alpha\right)$ is an unstable focus if

$$
\frac{\alpha^{2}+\beta+2 h \alpha^{2}+2 h^{2} \alpha^{2}\left(\alpha^{2}+\beta\right)}{(1+h)\left(\alpha^{2}+\beta\right)\left(1+h\left(\beta+\alpha^{2}\right)\right)}>1 .
$$

(iii) $P_{x y}^{+}\left(\alpha /\left(\beta+\alpha^{2}\right), \alpha\right)$ is nonhyperbolic if

$$
\frac{\alpha^{2}+\beta+2 h \alpha^{2}+2 h^{2} \alpha^{2}\left(\alpha^{2}+\beta\right)}{(1+h)\left(\alpha^{2}+\beta\right)\left(1+h\left(\beta+\alpha^{2}\right)\right)}=1 .
$$

Hereafter, we will establish that $P_{x y}^{+}\left(\alpha /\left(\beta+\alpha^{2}\right), \alpha\right)$ of (4) is the periodic point of prime period -1 . Moreover, equilibrium $P_{x y}^{+}\left(\alpha /\left(\beta+\alpha^{2}\right), \alpha\right)$ is the periodic point having period $-2,3, \ldots, n$.

\section{Existence of Prime Period and Periodic Points of (4)}

Theorem 1. $P_{x y}^{+}\left(\alpha /\left(\beta+\alpha^{2}\right), \alpha\right)$ of the model (4) is a periodic point of prime period -1 .

Proof. From (4), we define

$$
F(x, y)=(f(x, y), g(x, y)) \text {, }
$$

where

$$
\begin{aligned}
& f(x, y)=\frac{\alpha h+x}{1+h \beta+h y^{2}}, \\
& g(x, y)=\frac{y+h \beta x+h x y^{2}}{1+h} .
\end{aligned}
$$


After manipulation, from (19), one gets

$$
F_{P_{x y}^{+}\left(\alpha /\left(\beta+\alpha^{2}\right), \alpha\right)}=P_{x y}^{+}\left(\frac{\alpha}{\beta+\alpha^{2}}, \alpha\right) \text {. }
$$

From (21), one can conclude that $P_{x y}^{+}\left(\alpha /\left(\beta+\alpha^{2}\right), \alpha\right)$ of (4) is a periodic point of prime period -1 .
Theorem 2. $P_{x y}^{+}\left(\alpha /\left(\beta+\alpha^{2}\right), \alpha\right)$ of the glycolytic oscillator model (4) is a periodic point of period $-2,3, \ldots, n$.

Proof. From (19), one has

$$
\begin{aligned}
F^{2}(x, y) & =\left(\frac{\alpha h+f(x, y)}{1+h \beta+h(g(x, y))^{2}}, \frac{g(x, y)+h \beta f(x, y)}{1+h}+\frac{h\left(f(x, y) g(x, y)^{2}\right)}{1+h}\right) \\
& \Longrightarrow F_{P_{x y}^{+}\left(\alpha /\left(\beta+\alpha^{2}\right), \alpha\right)}^{2}=P_{x y}^{+}\left(\frac{\alpha}{\beta+\alpha^{2}}, \alpha\right), \\
F^{3}(x, y) & =\left(\frac{\alpha h+f^{2}(x, y)}{1+h \beta+h\left(g^{2}(x, y)^{2}\right)}, \frac{g^{2}(x, y)^{2}+h \beta f^{2}(x, y)}{1+h}+\frac{h\left(f^{2}(x, y)\left(g^{2}(x, y)\right)\right)^{2}}{1+h}\right) \\
& \Longrightarrow F_{P_{x y}^{+}\left(\alpha /\left(\beta+\alpha^{2}\right), \alpha\right)}^{3}=P_{x y}^{+}\left(\frac{\alpha}{\beta+\alpha^{2}}, \alpha\right), \\
F^{i}(x, y) & =\left(\frac{\alpha h+f^{i-1}(x, y)}{1+h \beta+h\left(g^{i-1}(x, y)^{2}\right)}, \frac{g^{i-1}(x, y)+h \beta f^{i-1}(x, y)}{1+h}+\frac{h\left(f^{i-1}(x, y) g^{i-1}(x, y)^{2}\right)}{1+h}\right) \\
& \Longrightarrow F_{P_{x y}^{i}\left(\alpha /\left(\beta+\alpha^{2}\right), \alpha\right)}^{i}=P_{x y}^{+}\left(\frac{\alpha}{\beta+\alpha^{2}}, \alpha\right) .
\end{aligned}
$$

From (22), $P_{x y}^{+}\left(\alpha /\left(\beta+\alpha^{2}\right), \alpha\right)$ of $(4)$ is a periodic point of period $-2,3, \ldots, n$.

\section{Bifurcation Analysis about} $P_{x y}^{+}\left(\alpha /\left(\beta+\alpha^{2}\right), \alpha\right)$ of the Glycolytic Oscillator Model (4)

Recall that if (18) holds, then $\left|\lambda_{1,2}\right|_{(18)}=1$, which implies that the glycolytic oscillator model (4) undergoes N-S bifurcation if parameters $h, \alpha$, and $\beta$ go through the curve:

$$
\mathrm{N}-\mathrm{SB}_{P_{x y}^{+}\left(\alpha /\left(\beta+\alpha^{2}\right), \alpha\right)}=\left\{(h, \alpha, \beta): \frac{\alpha^{2}+\beta+2 h \alpha^{2}+2 h^{2} \alpha^{2}\left(\alpha^{2}+\beta\right)}{(1+h)\left(\alpha^{2}+\beta\right)\left(1+h\left(\beta+\alpha^{2}\right)\right)}=1, h, \alpha, \beta>0\right\} .
$$

Hereafter, we will give detailed N-S bifurcation about $P_{x y}^{+}\left(\alpha /\left(\beta+\alpha^{2}\right), \alpha\right)$ when parameters $h, \alpha$, and $\beta$ go through the curve, which is depicted in (23), by using bifurcation theory $[7,8]$. For given parameters $h, \alpha$, and $\beta$, let

$$
\begin{aligned}
F(h, \alpha, \beta)= & \alpha^{2}+\beta+2 h \alpha^{2}+2 h^{2} \alpha^{2}\left(\alpha^{2}+\beta\right) \\
& -(1+h)\left(\alpha^{2}+\beta\right)\left(1+h\left(\beta+\alpha^{2}\right)\right) .
\end{aligned}
$$

After simplifying (24), one gets

$$
F(h, \alpha, \beta)=\alpha^{2}+h \alpha^{4}-\beta^{2}-2 \alpha^{2} \beta-\beta-\beta^{2} h-\alpha^{4} .
$$

From (25), one gets $\partial F(h, \alpha, \beta) / \partial \beta<0$. So, by implicit function theorem, one can obtain $\beta=\beta(h, \alpha)$ such that $F(h, \alpha, \beta(h, \alpha))=0$ and hence choose $\beta$ as a bifurcation parameter. Now, consider $\beta$ in a small neighborhood of $\beta^{*}$, i.e., $\beta=\beta^{*}+\varepsilon$, where $\varepsilon \ll 1$ and hence the glycolytic oscillator model (4) becomes

$$
\begin{aligned}
& x_{n+1}=\frac{\alpha h+x_{n}}{1+h\left(\beta^{*}+\varepsilon\right)+h y_{n}^{2}}, \\
& y_{n+1}=\frac{y_{n}+h\left(\beta^{*}+\varepsilon\right) x_{n}+h x_{n} y_{n}^{2}}{1+h},
\end{aligned}
$$


where $P_{x y}^{+}\left(\alpha /\left(\beta^{*}+\varepsilon+\alpha^{2}\right), \alpha\right)$ be its unique positive fixed point. Moreover, $\left.J\right|_{P_{x y}^{+}\left(\alpha /\left(\beta^{*}+\varepsilon+\alpha^{2}\right), \alpha\right)}$ about the fixed point $P_{x y}^{+}\left(\alpha /\left(\beta^{*}+\varepsilon+\alpha^{2}\right), \alpha\right)$ of $(26)$ is

$$
\left.J\right|_{P_{x y}^{+}\left(\alpha /\left(\beta^{*}+\varepsilon+\alpha^{2}\right), \alpha\right)}=\left(\begin{array}{cc}
\frac{1}{1+h\left(\beta^{*}+\varepsilon+\alpha^{2}\right)}-\frac{2 \alpha^{2} h}{\left(\alpha^{2}+\beta^{*}+\varepsilon\right)\left(1+h\left(\beta^{*}+\varepsilon+\alpha^{2}\right)\right)} \\
\frac{h\left(\beta^{*}+\varepsilon+\alpha^{2}\right)}{1+h} & \frac{\alpha^{2}+\beta^{*}+\varepsilon+2 h \alpha^{2}}{\left(\beta^{*}+\varepsilon+\alpha^{2}\right)(1+h)}
\end{array}\right) .
$$

The auxiliary equation of $\left.J\right|_{P_{x y}^{+}\left(\alpha /\left(\beta^{*}+\varepsilon+\alpha^{2}\right), \alpha\right)}$ about $P_{x y}^{+}\left(\alpha /\left(\beta^{*}+\varepsilon+\alpha^{2}\right), \alpha\right)$ is

where

$$
\begin{aligned}
& p\left(\frac{\alpha}{\beta^{*}+\varepsilon+\alpha^{2}}, \alpha\right)=\frac{(1+h)\left(\beta^{*}+\varepsilon+\alpha^{2}\right)+\left(\alpha^{2}+\beta^{*}+\varepsilon+2 h \alpha^{2}\right)\left(1+h\left(\alpha^{2}+\beta^{*}+\varepsilon\right)\right)}{\left(1+h\left(\alpha^{2}+\beta^{*}+\varepsilon\right)\right)(1+h)\left(\beta^{*}+\varepsilon+\alpha^{2}\right)}, \\
& q\left(\frac{\alpha}{\beta^{*}+\varepsilon+\alpha^{2}}, \alpha\right)=\frac{\alpha^{2}+\beta^{*}+\varepsilon+2 h \alpha^{2}+2 h^{2} \alpha^{2}\left(\alpha^{2}+\beta^{*}+\varepsilon\right)}{(1+h)\left(\alpha^{2}+\beta^{*}+\varepsilon\right)\left(1+h\left(\beta^{*}+\varepsilon+\alpha^{2}\right)\right)} .
\end{aligned}
$$

The zeroes of (28) are

$$
\begin{aligned}
\lambda_{1,2}= & \frac{p(\varepsilon) \pm \iota \sqrt{4 q(\varepsilon)-p^{2}(\varepsilon)}}{2} \\
= & \frac{\left((1+h)\left(\beta^{*}+\varepsilon+\alpha^{2}\right)+\left(\alpha^{2}+\beta^{*}+\varepsilon+2 h \alpha^{2}\right)\left(1+h\left(\alpha^{2}+\beta^{*}+\varepsilon\right)\right)\right) /\left(\left(1+h\left(\alpha^{2}+\beta^{*}+\varepsilon\right)\right)(1+h)\left(\beta^{*}+\varepsilon+\alpha^{2}\right)\right)}{2} \\
& \pm \frac{\iota}{2} \sqrt{\Delta^{*}},
\end{aligned}
$$

where

$$
\Delta^{*}=4\left(\frac{\alpha^{2}+\beta^{*}+\varepsilon+2 h \alpha^{2}+2 h^{2} \alpha^{2}\left(\alpha^{2}+\beta^{*}+\varepsilon\right)}{(1+h)\left(\alpha^{2}+\beta^{*}+\varepsilon\right)\left(1+h\left(\beta^{*}+\varepsilon+\alpha^{2}\right)\right)}\right)-\left(\frac{(1+h)\left(\beta^{*}+\varepsilon+\alpha^{2}\right)+\left(\alpha^{2}+\beta^{*}+\varepsilon+2 h \alpha^{2}\right)\left(1+h\left(\alpha^{2}+\beta^{*}+\varepsilon\right)\right)}{\left(1+h\left(\alpha^{2}+\beta^{*}+\varepsilon\right)\right)(1+h)\left(\beta^{*}+\varepsilon+\alpha^{2}\right)}\right)^{2},
$$

$$
\begin{aligned}
\left|\lambda_{1,2}\right| & =(q(\varepsilon))^{1 / 2}, \\
\left.\frac{\mathrm{d}\left|\lambda_{1,2}\right|}{\mathrm{d} \varepsilon}\right|_{\varepsilon=0} & \neq 0 .
\end{aligned}
$$

Additionally, it is required that $\lambda_{1,2}^{m} \neq 1, m=1, \ldots, 4$, which is equivalent to $p(0) \neq-2,0,1,2$, and hence by manipulation, it is true. If $u_{n}=x_{n}-x^{*}, v_{n}=y_{n}-y^{*}$, then $P_{x y}^{+}\left(\alpha /\left(\beta^{*}+\varepsilon+\alpha^{2}\right), \alpha\right)$ of the glycolytic oscillator model (26) transforms into $P_{00}(0,0)$. So

$$
\begin{aligned}
& u_{n+1}=\frac{\alpha h+u_{n}+x^{*}}{1+h\left(\beta^{*}+\varepsilon\right)+h\left(v_{n}+y^{*}\right)^{2}}-x^{*}, \\
& v_{n+1}=\frac{v_{n}+y^{*}+h\left(u_{n}+x^{*}\right)\left(\beta^{*}+\varepsilon+\left(v_{n}+y^{*}\right)^{2}\right)}{1+h}-y^{*},
\end{aligned}
$$


where $x^{*}=\alpha /\left(\beta+\alpha^{2}\right)$ and $y^{*}=\alpha$. Hereafter, the normal form of (33) is studied if $\varepsilon=0$. By Taylor series expansion about $\left(u_{n}, v_{n}\right)=(0,0)$, from (33), one gets

$$
\begin{aligned}
& u_{n+1}=a_{11} u_{n}+a_{12} v_{n}+a_{13} u_{n} v_{n}+a_{14} v_{n}^{2}+o\left(\left(\left|u_{n}\right|+\left|v_{n}\right|\right)^{2}\right), \\
& v_{n+1}=a_{21} u_{n}+a_{22} v_{n}+a_{23} u_{n} v_{n}+a_{24} v_{n}^{2}+o\left(\left(\left|u_{n}\right|+\left|v_{n}\right|\right)^{2}\right),
\end{aligned}
$$

where

$$
\begin{aligned}
& a_{11}=\frac{1}{1+h\left(\beta^{*}+y^{*^{2}}\right)}, \\
& a_{12}=\frac{-2 h y^{*}\left(x^{*}+h \alpha\right)}{1+h\left(\beta^{*}+y^{*^{2}}\right)}, \\
& a_{13}=\frac{-2 h y^{*}}{\left(1+h\left(\beta^{*}+y^{*^{2}}\right)\right)^{2}}, \\
& a_{14}=\frac{h\left(x^{*}+h \alpha\right)\left(-\left(1+h \beta^{*}+h y^{*^{2}}\right)+4 y^{*^{2}} h\right)}{\left(1+h\left(\beta^{*}+y^{*^{2}}\right)\right)^{3}}, \\
& a_{21}=\frac{h\left(\beta^{*}+y^{*^{2}}\right)}{1+h}, \\
& a_{22}=\frac{1+2 h x^{*} y^{*}}{1+h}, \\
& a_{23}=\frac{2 h y^{*}}{1+h}, \\
& a_{24}=\frac{h x^{*}}{1+h} .
\end{aligned}
$$

Hereafter, one contracts the following invertible matrix $T$ that puts the linear part of (34) into the canonical form:

$$
T:=\left(\begin{array}{cc}
a_{12} & 0 \\
\eta-a_{11} & -\zeta
\end{array}\right)
$$

where

$$
\begin{aligned}
& \eta=\frac{(1+h)\left(\beta+\alpha^{2}\right)+\left(\alpha^{2}+\beta+2 h \alpha^{2}\right)\left(1+h\left(\alpha^{2}+\beta\right)\right)}{2\left(1+h\left(\alpha^{2}+\beta\right)\right)(1+h)\left(\beta+\alpha^{2}\right)}, \\
& \zeta=\frac{1}{2} \sqrt{\Delta^{*}}
\end{aligned}
$$

and $\Delta^{*}$ is depicted in (31). Hence, (34) then implies

$$
\begin{aligned}
& X_{n+1}=\eta X_{n}-\zeta Y_{n}+\bar{P}, \\
& Y_{n+1}=\zeta X_{n}+\eta Y_{n}+\bar{Q},
\end{aligned}
$$

where

$$
\begin{aligned}
& \bar{P}\left(X_{n}, Y_{n}\right)=l_{11} X_{n}^{2}+l_{12} X_{n} Y_{n}+l_{13} y_{n}^{2}+o\left(\left(\left|X_{n}\right|+\left|Y_{n}\right|\right)^{2}\right), \\
& \bar{Q}\left(X_{n}, Y_{n}\right)=l_{21} X_{n}^{2}+l_{22} X_{n} Y_{n}+l_{23} y_{n}^{2}+o\left(\left(\left|X_{n}\right|+\left|Y_{n}\right|\right)^{2}\right),
\end{aligned}
$$

in which

$$
\begin{aligned}
& l_{11}=\frac{\eta-a_{11}}{a_{12}}\left[a_{12} a_{13}+a_{14}\left(\eta-a_{11}\right)\right], \\
& l_{12}=-\frac{\zeta}{a_{12}}\left[a_{12} a_{13}+2 a_{14}\left(\eta-a_{11}\right)\right], \\
& l_{13}=\frac{a_{14}}{a_{12}} \zeta^{2}, \\
& l_{21}=\frac{\eta-a_{11}}{\zeta a_{12}}\left[\left(\eta-a_{11}\right)\left(a_{12}\left(a_{13}-a_{24}\right)-a_{14} a_{24}\right)-a_{12}^{2} a_{23}\right], \\
& l_{22}=-\frac{\eta-a_{11}}{a_{12}}\left[2 a_{14}\left(\eta-a_{11}\right)+a_{12}\left(a_{13}+2 a_{24}\right)+a_{23} a_{12}^{2}\right], \\
& l_{23}=\frac{\zeta}{a_{12}}\left[a_{14} \zeta\left(\eta-a_{11}\right)-a_{12} a_{24}\right],
\end{aligned}
$$

by

$$
\left(\begin{array}{c}
u_{n} \\
v_{n}
\end{array}\right):=\left(\begin{array}{cc}
a_{12} & 0 \\
\eta-a_{11} & -\zeta
\end{array}\right)\left(\begin{array}{l}
X_{n} \\
Y_{n}
\end{array}\right) .
$$

From (39), one gets

$$
\begin{aligned}
& \left.\bar{P}_{X_{n} X_{n}}\right|_{P_{00}(0,0)}=2 l_{11}, \\
& \left.\bar{P}_{X_{n} Y_{n}}\right|_{P_{00}(0,0)}=l_{12}, \\
& \left.\bar{P}_{Y_{n} Y_{n}}\right|_{P_{00}(0,0)}=2 l_{13}, \\
& \left.\bar{Q}_{X_{n} X_{n}}\right|_{P_{00}(0,0)}=2 l_{21}, \\
& \left.\bar{Q}_{X_{n} Y_{n}}\right|_{P_{00}(0,0)}=l_{22}, \\
& \left.\bar{Q}_{Y_{n} Y_{n}}\right|_{P_{00}^{+}(0,0)}=2 l_{23} .
\end{aligned}
$$

In order to ensure the N-S bifurcation for (38), it is required that the following discriminatory quantity should be nonzero ([7-14]):

$$
\Omega=-\operatorname{Re}\left[\frac{(1-2 \bar{\lambda}) \bar{\lambda}^{2}}{1-\lambda} \xi_{11} \xi_{20}\right]-\frac{1}{2}\left\|\xi_{11}\right\|^{2}-\left\|\xi_{02}\right\|^{2}+\operatorname{Re}\left(\bar{\lambda} \xi_{21}\right),
$$


where

$$
\begin{aligned}
& \xi_{02}=\left.\frac{1}{8}\left[\bar{P}_{X_{n} X_{n}}-\bar{P}_{Y_{n} Y_{n}}+2 \bar{Q}_{X_{n} Y_{n}}+\iota\left(\bar{Q}_{X_{n} X_{n}}-\bar{Q}_{Y_{n} Y_{n}}+2 \bar{P}_{X_{n} Y_{n}}\right)\right]\right|_{P_{00}(0,0)}, \\
& \xi_{11}=\left.\frac{1}{4}\left[\bar{P}_{X_{n} X_{n}}+\bar{P}_{Y_{n} Y_{n}}+\iota\left(\bar{Q}_{X_{n} X_{n}}+\bar{Q}_{Y_{n} Y_{n}}\right)\right]\right|_{P_{00}(0,0)}, \\
& \xi_{20}=\left.\frac{1}{8}\left[\bar{P}_{X_{n} X_{n}}-\bar{P}_{Y_{n} Y_{n}}+2 \bar{Q}_{X_{n} Y_{n}}+\iota\left(\bar{Q}_{X_{n} X_{n}}-\bar{Q}_{Y_{n} Y_{n}}-2 \bar{P}_{X_{n} Y_{n}}\right)\right]\right|_{P_{00}(0,0)}, \\
& \xi_{21}=\left.\frac{1}{16}\left[\bar{P}_{X_{n} X_{n} X_{n}}+\bar{P}_{X_{n} Y_{n} Y_{n}}+\bar{Q}_{X_{n} X_{n} Y_{n}}+\bar{Q}_{Y_{n} Y_{n} Y_{n}}+\iota\left(\bar{Q}_{X_{n} X_{n} X_{n}}+\bar{Q}_{X_{n} Y_{n} Y_{n}}-\bar{P}_{X_{n} X_{n} Y_{n}}-P_{Y_{n} Y_{n} Y_{n}}\right)\right]\right|_{P_{00}(0,0)} .
\end{aligned}
$$

After manipulation, one gets

$$
\begin{aligned}
& \xi_{02}=\frac{1}{8}\left[2 l_{11}-l_{12}+2 l_{22}+2 \iota\left(l_{21}+l_{12}-l_{23}\right)\right], \\
& \xi_{11}=\frac{1}{2}\left[l_{11}+l_{13}+\iota\left(l_{21}+l_{23}\right)\right], \\
& \xi_{20}=\frac{1}{4}\left[l_{11}-l_{13}+l_{22}+\iota\left(l_{21}-l_{23}-l_{12}\right)\right], \\
& \xi_{21}=0 .
\end{aligned}
$$

From the analysis, one can conclude the following.

Theorem 3. If $\Omega \neq 0$, then the glycolytic oscillator model (4) undergoes N-S bifurcation about the fixed point: $P_{x y}^{+}\left(\alpha /\left(\beta+\alpha^{2}\right), \alpha\right)$ as parameters $h, \alpha$, and $\beta$ go through the curve, which is depicted in (23). Additionally, attracting (resp., repelling) closed curve bifurcates appear from $P_{x y}^{+}\left(\alpha /\left(\beta+\alpha^{2}\right), \alpha\right)$ if $\Omega<0$ (resp., $\Omega>0$ ).

According to bifurcation theory, the bifurcation is supercritical (resp., subcritical) N-S bifurcation if $\Omega<0$ (resp., $\Omega>0$ ). In the subsequent section, simulations guarantee that the glycolytic oscillator model (4) undergoes the supercritical $N$-S bifurcation if $h, \alpha$, and $\beta$ go through (23).

Remark. The discrete-time glycolytic oscillator model (4) cannot undergo any bifurcation, except N-S bifurcation, about the positive fixed point $P_{x y}^{+}\left(\alpha /\left(\beta+\alpha^{2}\right), \alpha\right)$.

\section{Numerical Simulations}

Theoretical results are verified numerically in this section. For instance, if $\alpha=0.6, h=0.7$, then from (18), one gets $\beta=$ 0.16105922820694768 . Theoretically, equilibrium $P_{x y}^{+}(\alpha /(\beta$ $\left.+\alpha^{2}\right), \alpha$ ) of the glycolytic oscillator model (4) is a locally asymptotically stable focus if $\beta>0.16105922820694768$, i.e., condition (16) holds. To see this, if $\beta=0.4>$
0.16105922820694768 , then the unique positive equilibrium point $P_{x y}^{+}(0.7894736842105263,0.6)$ of $(4)$ is a locally asymptotically stable focus (see Figure 1(a)). Similarly for other values of $\beta$, if $\beta>0.16105922820694768$, then $P_{x y}^{+}\left(\alpha /\left(\beta+\alpha^{2}\right), \alpha\right)$ of (4) is a locally asymptotically stable focus (see Figures 1(b)-1(l)). But if $\beta<0.16105922820694768$, then positive equilibrium $P_{x y}^{+}\left(\alpha /\left(\beta+\alpha^{2}\right), \alpha\right)$ becomes an unstable focus, i.e., condition (17) holds. Precisely, if $\beta<0.16105922820694768$, then positive equilibrium $P_{x y}^{+}(\alpha /$ $\left.\left(\beta+\alpha^{2}\right), \alpha\right)$ loses its stability and meanwhile an attracting closed curve appears. The appearance of this closed curve indicates that the glycolytic oscillator model (4) undergoes a supercritical N-S bifurcation if $\alpha, \beta$, and $h$ vary in a neighborhood of $P_{x y}^{+}\left(\alpha /\left(\beta+\alpha^{2}\right), \alpha\right)$. To see this, if $\beta=0.16<0.16105922820694768$, then eigenvalues of $\left.J\right|_{P_{x y}^{+}\{(1.1538461538461537,0.6)\}}$ about $(1.1538461538461537,0.6)$ are

$$
\lambda_{1,2}=0.615799684186781 \pm 0.3270196350217114 \iota .
$$

After some manipulation, from (43), one gets

$$
\begin{aligned}
& \xi_{02}=-0.007611881686547611+0.024203687145576566 \iota, \\
& \xi_{11}=0.03740896019401933-0.1966375637334364 l, \\
& \xi_{20}=0.009013748156314753-0.0593513169940678 l, \\
& \xi_{21}=0 .
\end{aligned}
$$

In view of (46) and (47), from (43), one gets $\Omega=-0.029495873864781315<0$. Hence, if $\beta=0.16<$ 0.16105922820694768 , then model (4) undergoes a supercritical N-S bifurcation and so a stable curve appears, which is depicted in Figure 2(a). Also for different choices of the bifurcation parameter, if $\beta<0.16105922820694768$, then one gets $\Omega<0$ (see Table 2), and corresponding closed curves are depicted in Figures 2(b)-2(l). Moreover, the bifurcation diagrams along with the maximum Lyapunov exponent are plotted and drawn in Figure 3. Finally, 3D bifurcation diagrams are plotted and drawn in Figure 4. 


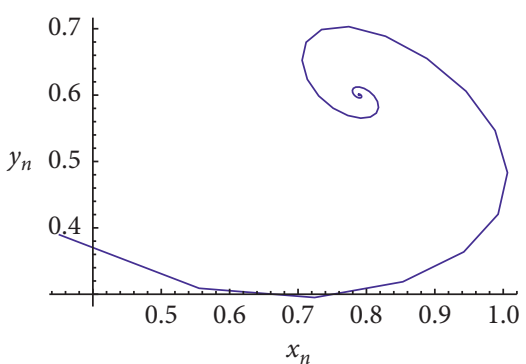

(a)

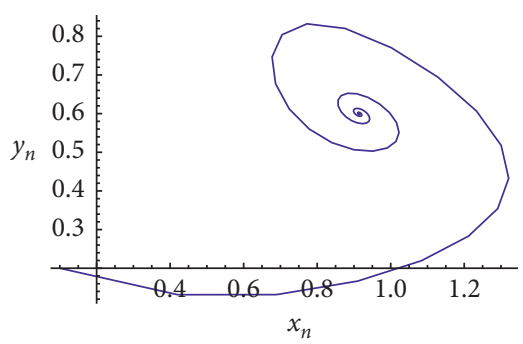

(d)

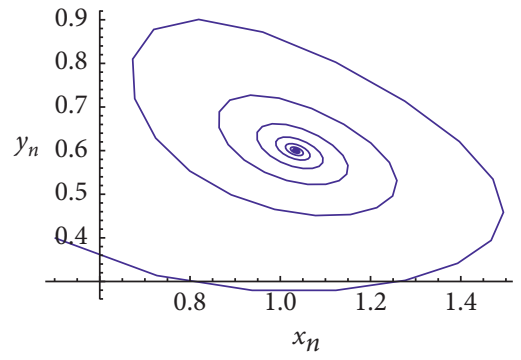

(g)

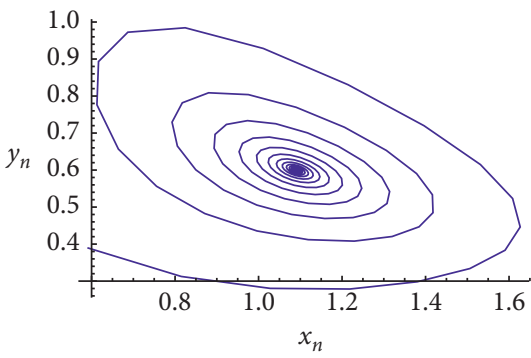

(j)

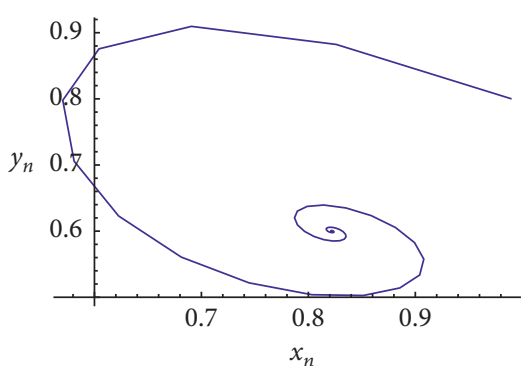

(b)

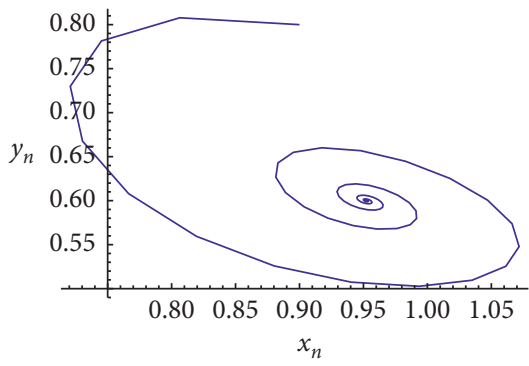

(e)

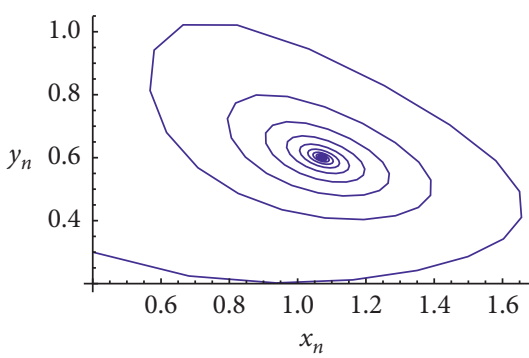

(h)

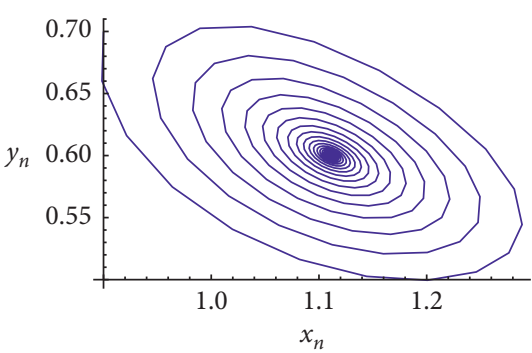

(k)

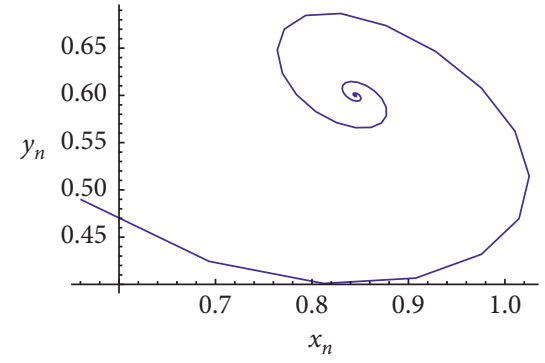

(c)

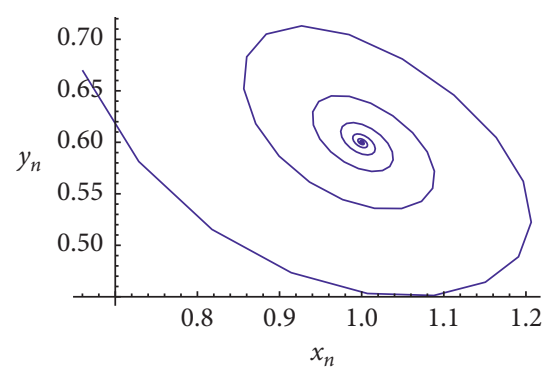

(f)

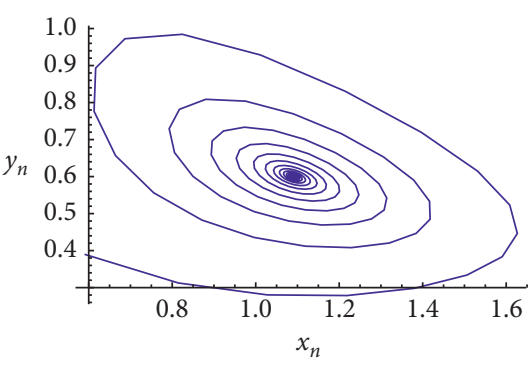

(i)

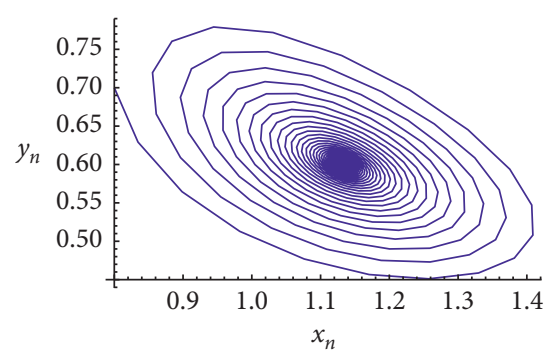

(l)

Figure 1: Trajectories of the discrete-time glycolytic oscillator model (4) about $P_{x y}^{+}\left(\alpha /\left(\beta+\alpha^{2}\right), \alpha\right)$ : (a) $\beta=0.4$ with $(0.35,0.39)$; (b) $\beta=0.37$ with $(0.99,0.80)$; (c) $\beta=0.35$ with $(0.56,0.49)$; (d) $\beta=0.2965$ with $(0.1,0.2)$; (e) $\beta=0.27$ with $(0.9,0.8)$; (f) $\beta=0.24$ with (0.66, 0.67); (g) $\beta=0.22$ with $(0.5,0.4)$; (h) $\beta=0.2$ with $(0.4,0.3)$; (i) $\beta=0.19$ with $(0.59,0.39)$; (j) $\beta=0.199$ with $(0.9,0.7)$; (k) $\beta=0.18$ with (0.9, 0.7); (l) $\beta=0.17$ with $(0.8,0.7)$.

\section{Control of N-S Bifurcation about $P_{x y}^{+}\left(\alpha /\left(\beta+\alpha^{2}\right), \alpha\right)$ of the Glycolytic Oscillator Model (4)}

The hybrid control strategy is applied to control N-S bifurcation about $P_{x y}^{+}\left(\alpha /\left(\beta+\alpha^{2}\right), \alpha\right)$ of the glycolytic oscillator model (4) motivated from the work of [15-18]. The control system corresponding to the glycolytic oscillator model (4) is

$$
\begin{aligned}
& x_{n+1}=c\left(\frac{\alpha h+x_{n}}{1+h \beta+h y_{n}^{2}}\right)+(1-c) x_{n}, \\
& y_{n+1}=c\left(\frac{y_{n}+h \beta x_{n}+h x_{n} y_{n}^{2}}{1+h}\right)+(1-c) y_{n} .
\end{aligned}
$$

$\left.J\right|_{P_{x y}^{+}\left(\alpha /\left(\beta+\alpha^{2}\right), \alpha\right)}$ about $P_{x y}^{+}\left(\alpha /\left(\beta+\alpha^{2}\right), \alpha\right)$ of the controlled system, which is depicted in (48), is 


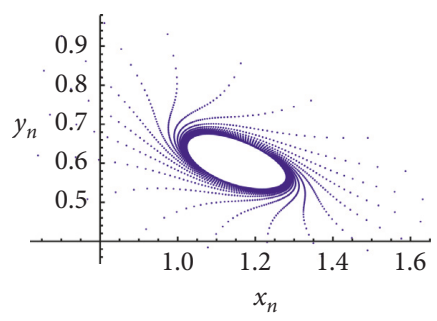

(a)

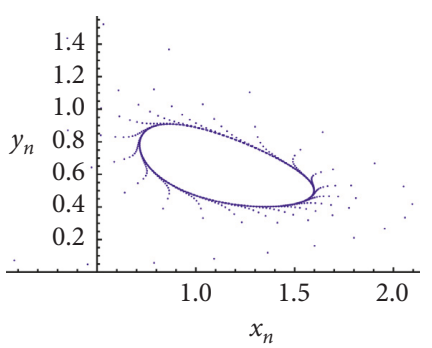

(d)

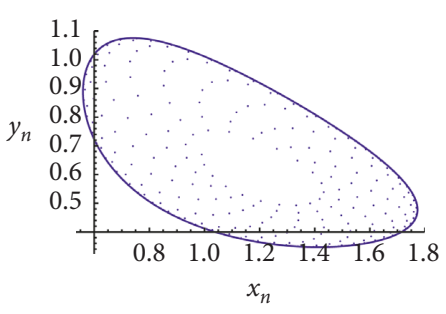

(g)

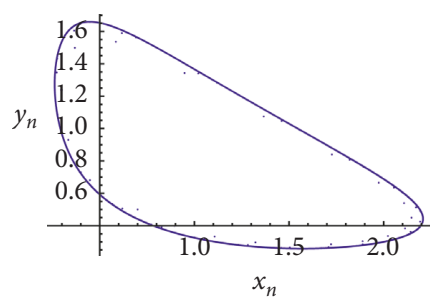

(j)

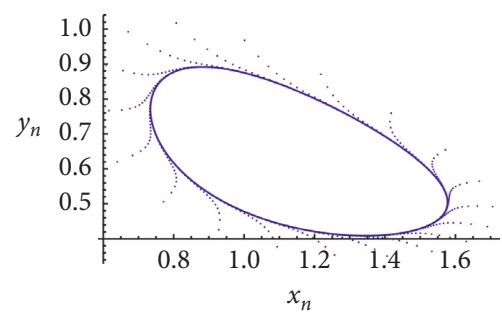

(b)

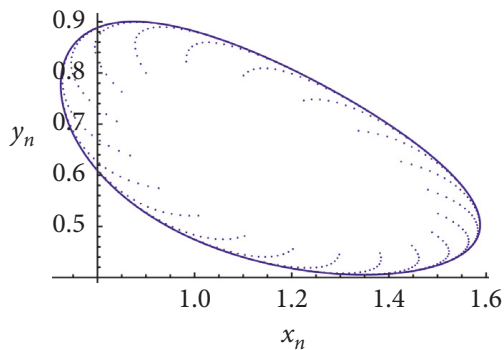

(e)

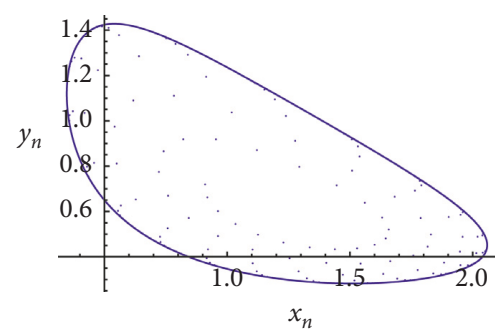

(h)

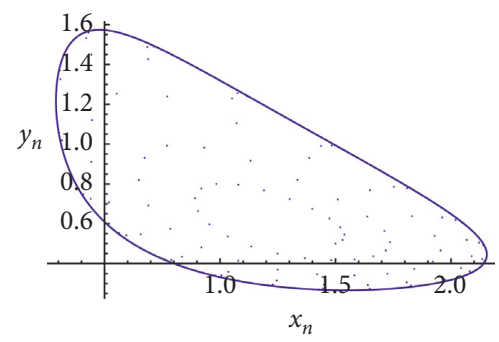

(k)

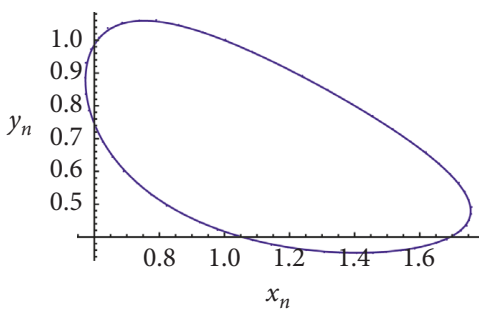

(c)

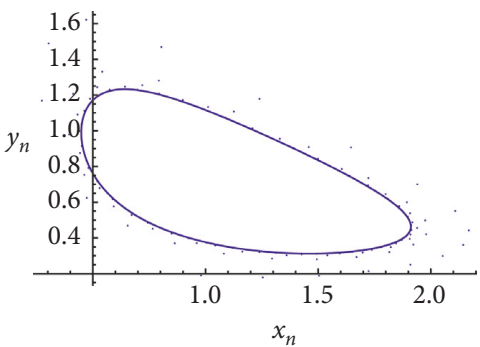

(f)

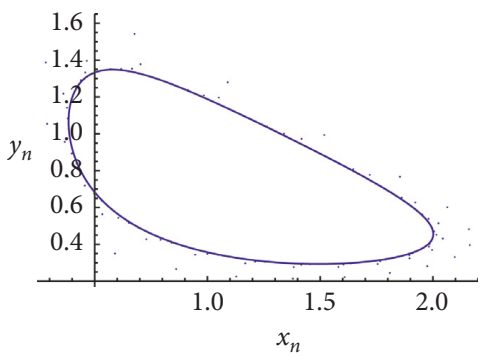

(i)

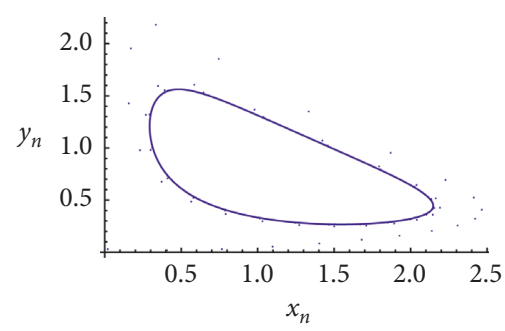

(1)

Figure 2: Supercritical N-S bifurcation of the discrete-time glycolytic oscillator model (4) about $P_{x y}^{+}\left(\alpha /\left(\beta+\alpha^{2}\right), \alpha\right):(\mathrm{a}) \beta=0.16$ with $(0.64$, $0.62)$; (b) $\beta=0.15$ with $(0.64,0.62)$; (c) $\beta=0.14$ with $(0.69,0.6)$; (d) $\beta=0.149$ with $(0.081,0.072)$; (e) $h=0.1495$ with $(0.9,0.8)$; (f) $\beta=0.13$ with (0.98, 0.19); (g) $\beta=0.139$ with $(0.99,0.67)$; (h) $\beta=0.12$ with $(0.91,0.72)$; (i) $\beta=0.1239$ with $(0.59,0.35)$; (j) $\beta=0.11$ with (0.7, 0.5); (k) $\beta=0.1135$ with $(1.5,0.5)$; (l) $\beta=0.114$ with $(0.02,0.03)$.

$$
\left.J\right|_{P_{x y}^{+}\left(\alpha /\left(\beta+\alpha^{2}\right), \alpha\right)}=\left(\begin{array}{cc}
\frac{c}{1+h \beta+h \alpha^{2}}+1-c & \frac{-2 h c \alpha^{2}}{\left(\alpha^{2}+\beta\right)\left(1+h\left(\beta+y^{2}\right)\right)^{2}} \\
\frac{h c\left(\alpha^{2}+\beta\right)}{1+h} & \frac{c\left(\alpha^{2}+\beta+2 h \alpha^{2}\right)}{(1+h)\left(\beta+\alpha^{2}\right)}+1-c
\end{array}\right) .
$$

The rest of this section deals with the study of N-S bifurcation of the controlled system (48) at $h=0.7$, $\beta=0.1601<0.16105922820694768$, and $\alpha=0.6$. In this case, (49) becomes

$$
\left.J\right|_{P_{x y}^{+}(1.15362,0.6)}=\left(\begin{array}{cc}
1-0.2669 c & -0.710407 c \\
0.214159 c & 1+0.158261 c
\end{array}\right),
$$

whose auxiliary equation is 
TABLE 2: Numerical values of $\Omega$ if $\beta<0.16105922820694768$.

\begin{tabular}{lc}
\hline Value of $\beta$ if $q>1$ & Numerical value of $\Omega$ \\
\hline 0.16 & $-0.029495873864781315<0$ \\
0.15 & $-0.024558009135300485<0$ \\
0.14 & $-0.025661100529415302<0$ \\
0.149 & $-0.02516944528172212<0$ \\
0.1495 & $-0.02461170028243595<0$ \\
0.13 & $-0.026828812841863014<0$ \\
0.139 & $-0.025774885406930285<0$ \\
0.12 & $-0.028066167307452507<0$ \\
0.1239 & $-0.027574969477341914<0$ \\
0.11 & $-0.029378827673349428<0$ \\
0.1135 & $-0.028910444972707013<0$ \\
0.114 & $-0.028844333111783357<0$ \\
\hline
\end{tabular}

$$
\lambda^{2}-p \lambda+q=0
$$

where $p=2-0.108492 c$ and $q=1-1.150755 c+1.152135 c^{2}$. In view of Lemma 1 of [15], one gets

$$
\begin{array}{r}
1-(2-0.108492 c)+1-1.150755 c+1.152135 c^{2}>0, \\
1-1.150755 c+1.152135 c^{2}=1, \\
(2-0.108492 c)^{2}-4\left(1-1.150755 c+1.152135 c^{2}\right)<0 .
\end{array}
$$

Let $c=0.998$, then from (52), one gets

$$
\begin{aligned}
1-(2-0.108492 c)+1-1.150755 c+1.152135 c^{2}=0.107354 & >0 \\
1-(1.150755) c+(1.152135) c^{2} & =1, \\
(2-(0.108492) c)^{2}-4\left(1-(1.150755) c+(1.152135) c^{2}\right) & =-0.417687<0
\end{aligned}
$$

which hold true. Also, $\left.J\right|_{P_{x y}^{+}(1.15362,0.6)}=(1.15362,0.6)$ in terms of the bifurcation parameter $\beta$ becomes

$$
\left.J\right|_{P_{x y}^{+}\left(\alpha /\left(\beta+\alpha^{2}\right), \alpha\right)}=\left(\begin{array}{cc}
0.002+\frac{0.998}{0.252+0.7 \beta} & -\frac{0.83832(0.6+0.42(0.36+\beta))}{(1.252+0.7 \beta)^{2}(0.36+\beta)} \\
0.410941(0.36+\beta) & 0.002+\frac{0.587059(0.864+\beta)}{0.36+\beta}
\end{array}\right)
$$

whose roots are

$$
\begin{aligned}
\lambda_{1,2}= & 1.69341+3.55991 \beta+0.70336 \beta^{2} \\
& -\frac{\sqrt{\Delta^{* *}}}{2\left(0.766224+2.5568 \beta+1.19 \beta^{2}\right)},
\end{aligned}
$$

where

$$
\begin{aligned}
\Delta^{* *}= & \left(-1.69341-3.55991 \beta-0.70336 \beta^{2}\right)^{2} \\
& -4\left(0.224208-1.2023 \beta-1.1886 \beta^{2}\right) \\
& \cdot\left(0.766224+2.5568 \beta+1.19 \beta^{2}\right) .
\end{aligned}
$$

If $\beta=0.1601$, then from (32), one gets

$$
\left.\frac{\mathrm{d}\left|\lambda_{1,2}\right|}{\mathrm{d} \varepsilon}\right|_{\varepsilon=0}=3.82461>0 .
$$

From (57), the nondegenerate condition holds, and hence, (48) undergoes N-S bifurcation at $\beta=0.1601$. In terms of above chosen parametric values: $\beta=0.1601, \alpha=0.6, h=0.7$, and $c=0.998$, the controlled system (48) becomes

$$
\begin{aligned}
& x_{n+1}=0.002 x_{n}+\frac{\left(0.41916+0.998 x_{n}\right)}{\left(1.11207+0.7 y_{n}^{2}\right)}, \\
& y_{n+1}=0.002 y_{n}+0.588235\left(0.6986 x_{n}+0.998 y_{n}+0.7 x_{n} y_{n}^{2}\right),
\end{aligned}
$$

whose fixed point is $P_{x y}^{+}(1.15362,0.6)$. Now, we transform $P_{x y}^{+}(1.15362,0.6)$ into $P_{00}(0,0)$ using the following transformation:

$$
\begin{aligned}
& u_{n}=x_{n}-1.15362, \\
& v_{n}=y_{n}-0.6
\end{aligned}
$$

where (58) becomes

$$
\begin{aligned}
u_{n+1}= & 0.733634 u_{n}-0.967106 v_{n}-0.450543 u_{n} v_{n}-0.154225 v_{n}^{2} \\
& +o\left(\left(\left|u_{n}\right|+\left|v_{n}\right|\right)^{2}\right), \\
v_{n+1}= & 0.213731 u_{n}+1.15794 v_{n}+0.493129 u_{n} v_{n}+0.474072 v_{n}^{2} \\
& +o\left(\left(\left|u_{n}\right|+\left|v_{n}\right|\right)^{2}\right) .
\end{aligned}
$$




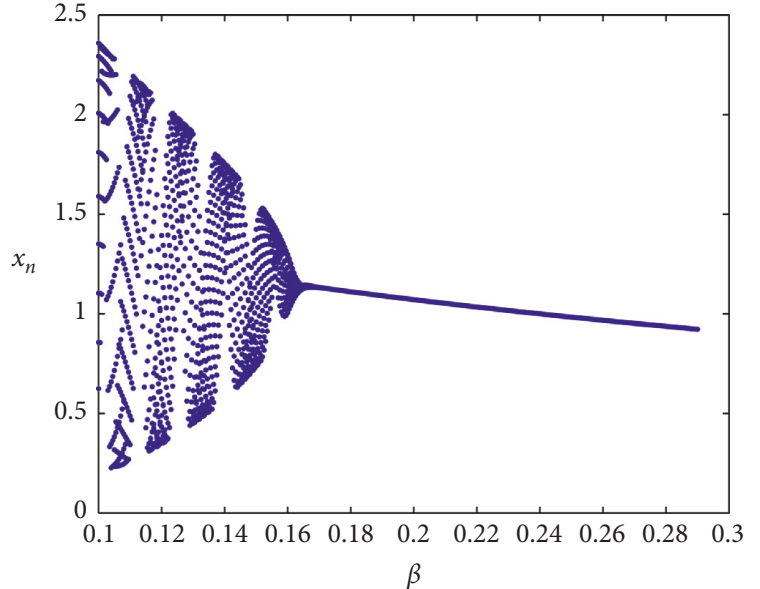

(a)

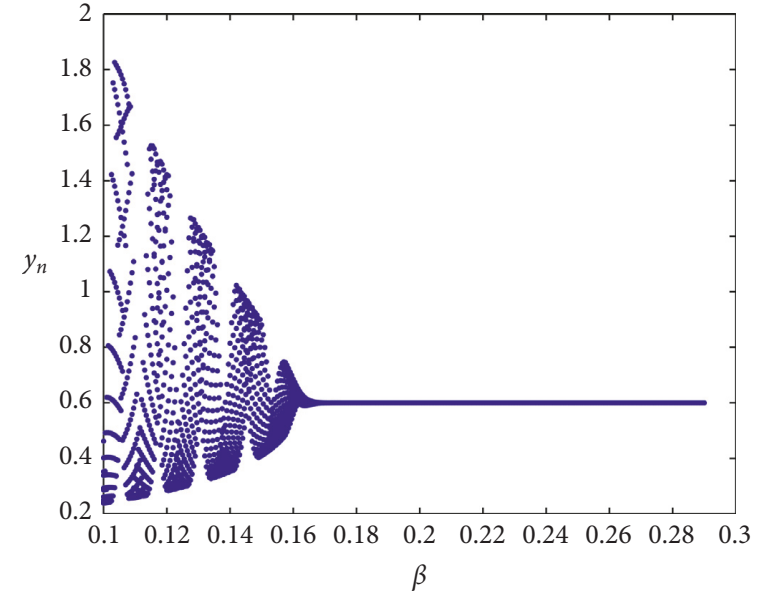

(b)

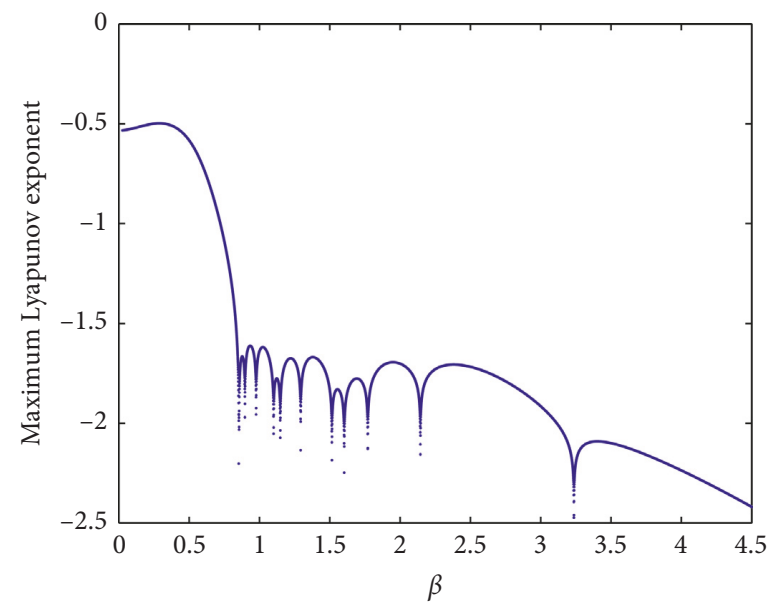

(c)

FiguRe 3: Bifurcation diagram and its corresponding maximum Lyapunov exponent of the model (4) about $P_{x y}^{+}\left(\alpha /\left(\beta+\alpha^{2}\right), \alpha\right)$. (a, b) Bifurcation diagram of the model if $\beta \in[0.1,0.29]$ and initial condition $(0.74,0.62)$. (c) Maximum Lyapunov exponent corresponding to (a) and (b).

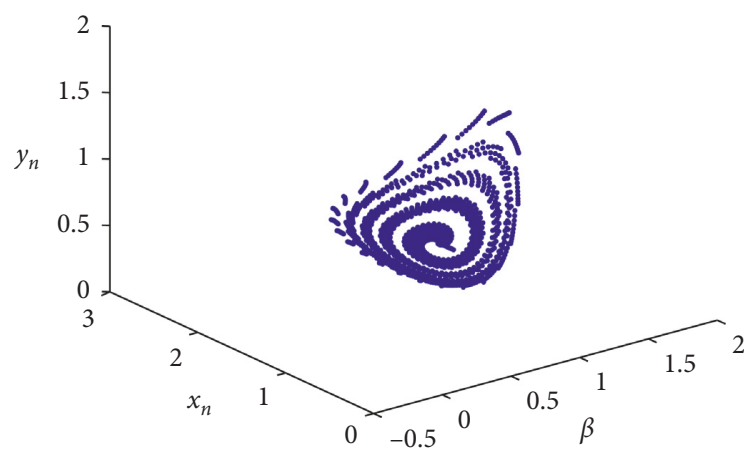

(a)

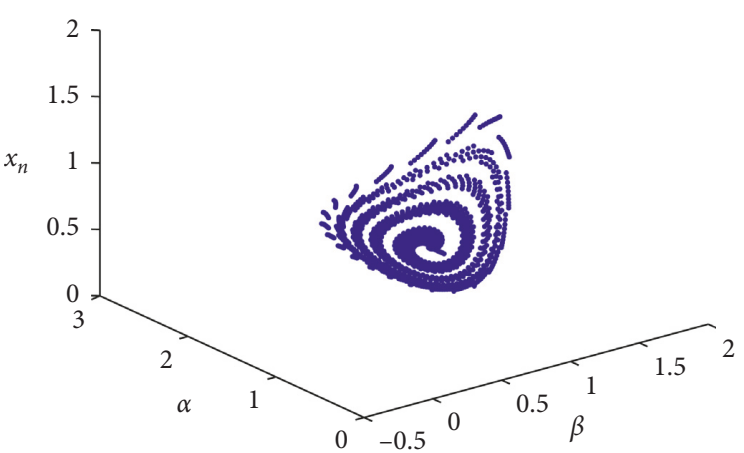

(b)

Figure 4: Continued. 


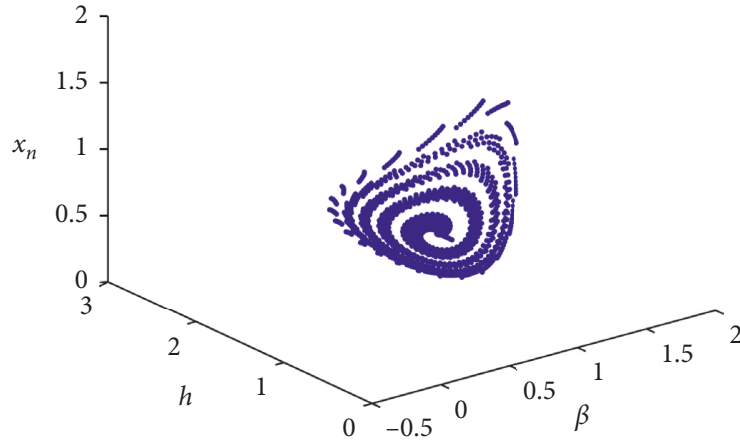

(c)

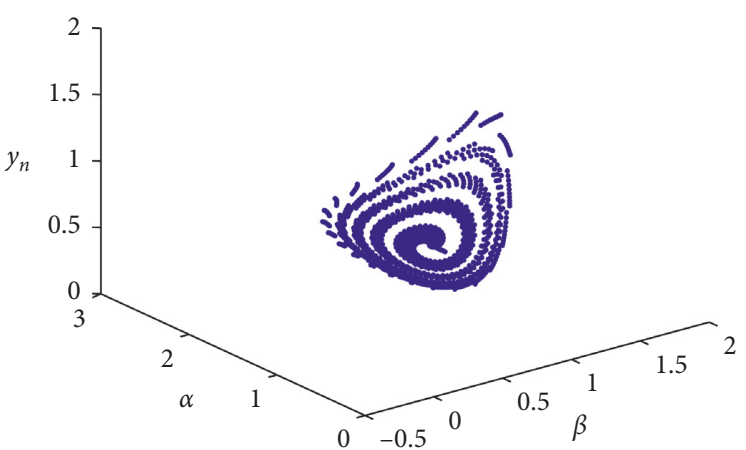

(d)

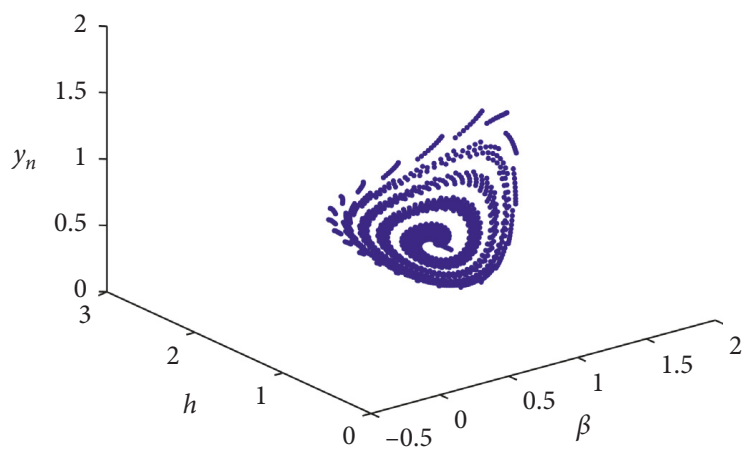

(e)

FIGURE 4: 3D bifurcation diagrams of the model (4).

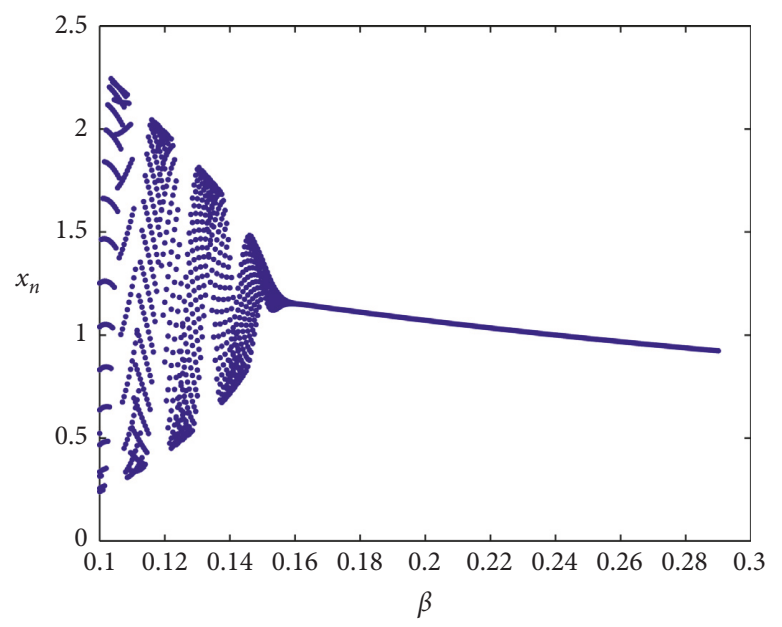

(a)

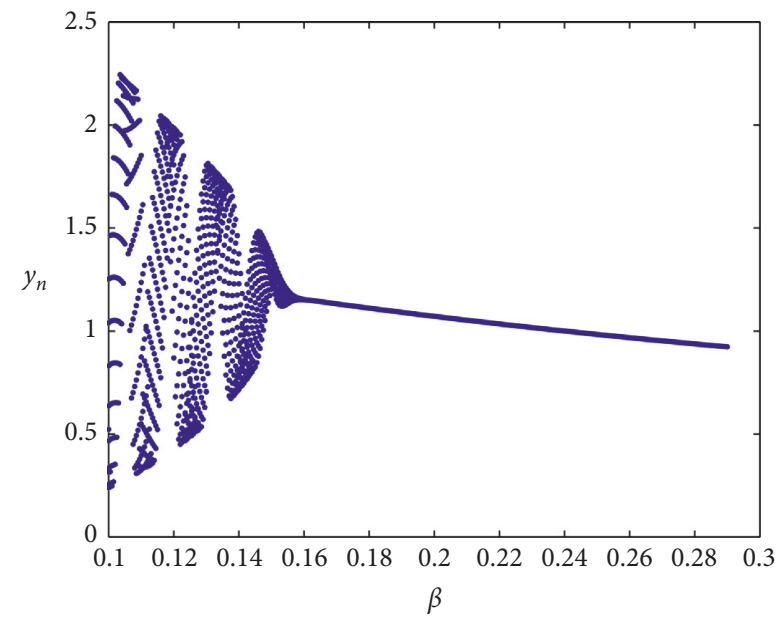

(b)

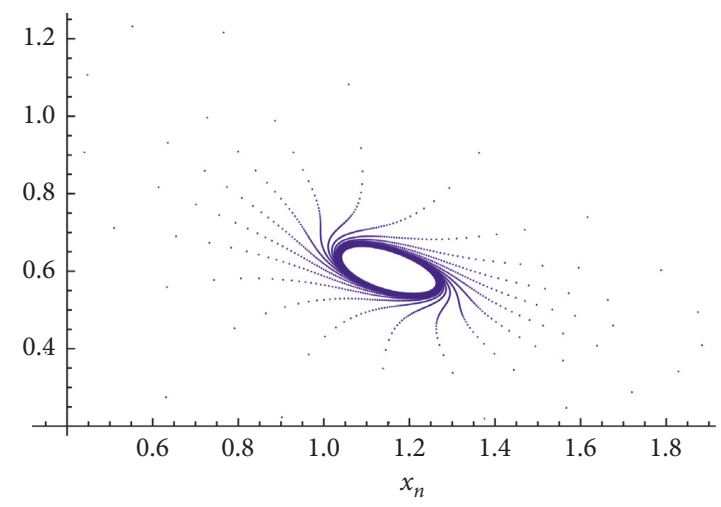

(c)

FiguRE 5: Control results of N-S bifurcation of the glycolytic oscillator model (4) about $P_{x y}^{+}\left(\alpha /\left(\beta+\alpha^{2}\right), \alpha\right)$ if $\alpha=0.6, h=0.7, c=0.998$, and $\beta \in[0.1,0.29]$. 


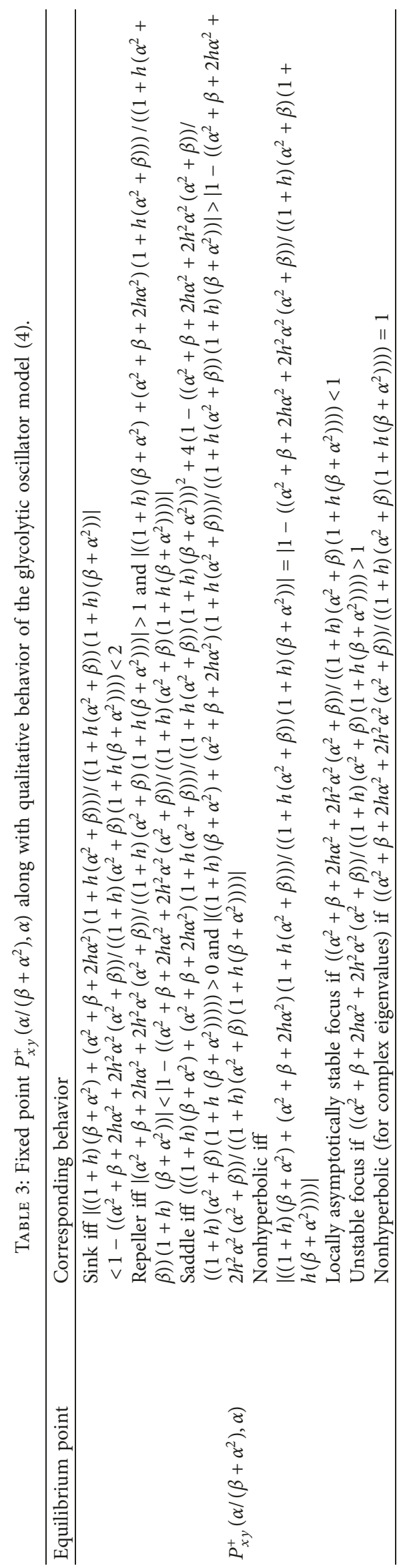


Since the linear part of $(60)$ is just as $\left.J\right|_{P_{x y}^{+}\left(\alpha /\left(\beta+\alpha^{2}\right), \alpha\right)}=$ $(1.15362,0.6)$ with eigenvalues,

$\lambda_{1,2}=0.6185299315830337 \pm 0.4140823777730747$.

Now, (61) takes the following form:

$$
\begin{aligned}
& \left(\begin{array}{c}
X_{n+1} \\
Y_{n+1}
\end{array}\right)=\left(\begin{array}{ll}
0.6185299315830337 & -0.4140823777730747 \\
0.4140823777730747 & -0.6185299315830337
\end{array}\right) \\
& \cdot\left(\begin{array}{c}
X_{n} \\
Y_{n}
\end{array}\right)+\left(\begin{array}{c}
\bar{P}\left(X_{n}, Y_{n}\right) \\
\bar{Q}\left(X_{n}, Y_{n}\right)
\end{array}\right)
\end{aligned}
$$

where

$$
\begin{aligned}
\bar{P}\left(X_{n}, Y_{n}\right)= & 0.0539722 X_{n}^{2}+0.201764 X_{n} Y_{n} \\
& +0.0273435 y_{n}^{2}+o\left(\left(\left|X_{n}\right|+\left|Y_{n}\right|\right)^{2}\right), \\
\bar{Q}\left(X_{n}, Y_{n}\right)= & -0.17732 X_{n}-0.00184395 X_{n} Y_{n} \\
& -0.203906 y_{n}^{2}+o\left(\left(\left|X_{n}\right|+\left|Y_{n}\right|\right)^{2}\right),
\end{aligned}
$$

by

$$
\begin{aligned}
\left(\begin{array}{l}
u_{n} \\
v_{n}
\end{array}\right):= & \left(\begin{array}{cc}
-0.9671063257065947 & 0 \\
-0.11510407546939039 & -0.4140823777730747
\end{array}\right) \\
& \cdot\left(\begin{array}{c}
X_{n} \\
Y_{n}
\end{array}\right) .
\end{aligned}
$$

In view of (63), from (45), one gets

$$
\begin{aligned}
& \xi_{02}=-0.012188390118933261+0.05708718392199485 \iota, \\
& \xi_{11}=0.04065784974095253-0.190612959926066 \iota, \\
& \xi_{20}=0.006196180814291302-0.04379461343161802 \iota, \\
& \xi_{21}=0 .
\end{aligned}
$$

In view of (61) and (65), from (43), one gets $\Omega=-0.028844333111783357<0$. Finally, the controlled results of N-S bifurcation are plotted and drawn in Figure 5. For more interesting results for solving nonlinear equations using the multipoint iterative method, we refer the reader to [19] and references cited therein.

\section{Conclusion}

In the proposed work, we have explored the local dynamics, $\mathrm{N}-\mathrm{S}$ bifurcation, and hybrid control in a discrete-time glycolytic oscillator model, which is depicted in (4), in the interior of $\mathbb{R}_{+}^{2}$. It is proved that $\forall \alpha$ and $\beta$, the glycolytic oscillator model (4) has a unique +ve equilibrium point $P_{x y}^{+}\left(\alpha /\left(\beta+\alpha^{2}\right), \alpha\right)$. By the method of linearization, we have explored the local dynamics along with topological classification about $P_{x y}^{+}\left(\alpha /\left(\beta+\alpha^{2}\right), \alpha\right)$ of the discrete-time glycolytic oscillator model (4), and conclusions are presented in Table 3. We have also investigated the prime period and periodic points for the model (4). Furthermore, we have explored the existence of bifurcation about $P_{x y}^{+}(\alpha /(\beta+$ $\left.\alpha^{2}\right), \alpha$ ) and proved that the glycolytic oscillator model (4) undergoes no bifurcation, except N-S bifurcation, when $h, \alpha$, and $\beta$ go through the curve $\mathrm{N}-\mathrm{SB}_{P_{x y}^{+}}\left(\alpha /\left(\beta+\alpha^{2}\right), \alpha\right)=$ $\left\{(h, \alpha, \beta):\left(\alpha^{2}+\beta+2 h \alpha^{2}+2 h^{2} \alpha^{2}\left(\alpha^{2}+\beta\right)\right) /\left((1+h)\left(\alpha^{2}+\beta\right)\right.\right.$ $\left.\left.\left(1+h\left(\beta+\alpha^{2}\right)\right)\right)=1\right\}$. We have performed the N-S bifurcation about $P_{x y}^{+}\left(\alpha /\left(\beta+\alpha^{2}\right), \alpha\right)$ by bifurcation theory. Moreover, the hybrid control strategy is applied to control the N-S bifurcation in the glycolytic oscillator model (4). Finally, theoretical results are verified numerically.

\section{Data Availability}

All the data utilized in this article have been included, and the sources from where they were adopted were cited accordingly.

\section{Conflicts of Interest}

The authors declare that they have no conflicts of interest.

\section{Acknowledgments}

A. Q. Khan and E. Abdullah research was partially supported by the Higher Education Commission of Pakistan, while the research of Tarek F. Ibrahim was funded by a Deanship of Scientific Research in King Khalid University under grant number GRP-324-40.

\section{References}

[1] R. H. Garrett and C. M. Grisham, Biochemistry, Cengage Learning. Inc., Boston, MA, USA, 1999.

[2] J. M. Berg, J. L. Tymoczko, and L. Stryer, Biochemistry, Macmillan, New York, NY, USA, 2002.

[3] T. Dandekar, S. Schuster, B. Snel, M. Huynen, and P. Bork, "Pathway alignment: application to the comparative analysis of glycolytic enzymes," Biochemical Journal, vol. 343, no. 1, pp. 115-124, 1999.

[4] A. Boiteux and B. Hess, "Design of glycolysis," Philosophical Transactions of the Royal Society B: Biological Sciences, vol. 293, no. 1063, pp. 5-22, 1981.

[5] L. E. Keshet, Mathematical Models in Bioilogy, Siam, Philadelphia, PA, USA, 1988.

[6] M. R. S. Kulenović and G. Ladas, Dynamics of Second-Order Rational Difference Equations: With Open Problems and Conjectures, Chapman and Hall/CRC, Boca Raton, FL, USA, 2002.

[7] J. Guckenheimer and P. Holmes, Nonlinear Oscillations, Dynamical Systems and Bifurcation of Vector Fields, SpringerVerlag, New York, NY, USA, 1983.

[8] Y. A. Kuznetsov, Elements of Applied Bifurcation Theorey, Springer-Verlag, New York, NY, USA, 3rd edition, 2004.

[9] X. Liu and D. Xiao, "Complex dynamic behaviors of a discrete-time predator-prey system," Chaos, Solitons \& Fractals, vol. 32, no. 1, pp. 80-94, 2007.

[10] A. Q. Khan, J. Ma, and D. Xiao, "Bifurcations of a two-dimensional discrete time plant-herbivore system," Communications in Nonlinear Science and Numerical Simulation, vol. 39, pp. 185-198, 2016.

[11] Z. Hu, Z. Teng, and L. Zhang, "Stability and bifurcation analysis of a discrete predator-prey model with 
nonmonotonic functional response," Nonlinear Analysis: Real World Applications, vol. 12, no. 4, pp. 2356-2377, 2011.

[12] Z. Jing and J. Yang, "Bifurcation and chaos in discrete-time predator-prey system," Chaos, Solitons \& Fractals, vol. 27, no. 1, pp. 259-277, 2006.

[13] C.-H. Zhang, X.-P. Yan, and G.-H. Cui, "Hopf bifurcations in a predator-prey system with a discrete delay and a distributed delay," Nonlinear Analysis: Real World Applications, vol. 11, no. 5, pp. 4141-4153, 2010.

[14] M. Sen, M. Banerjee, and A. Morozov, "Bifurcation analysis of a ratio-dependent prey-predator model with the Allee effect," Ecological Complexity, vol. 11, pp. 12-27, 2012.

[15] L.-G. Yuan and Q.-G. Yang, "Bifurcation, invariant curve and hybrid control in a discrete-time predator-prey system," Applied Mathematical Modelling, vol. 39, no. 8, pp. 23452362, 2015.

[16] Z. Chen and P. Yu, "Controlling and anti-controlling hopf bifurcations in discrete maps using polynomial functions," Chaos, Solitons \& Fractals, vol. 26, no. 4, pp. 1231-1248, 2005.

[17] E. M. Elabbasy, H. N. Agiza, H. El-Metwally, and A. A. Elsadany, "Bifurcation analysis, chaos and control in the burgers mapping," International Journal of Nonlinear Science, vol. 4, no. 3, pp. 171-185, 2007.

[18] G. Chen, J.-Q. Fang, Y. Hong, and H. Qin, "Controlling hopf bifurcations: discrete-time systems," Discrete Dynamics in Nature and Society, vol. 5, no. 1, pp. 29-33, 2000.

[19] M. Salimi, N. M. A. Nik Long, S. Sharifi, and B. A. Pansera, "A multi-point iterative method for solving nonlinear equations with optimal order of convergence," Japan Journal of Industrial and Applied Mathematics, vol. 35, no. 2, pp. 497-509, 2018. 


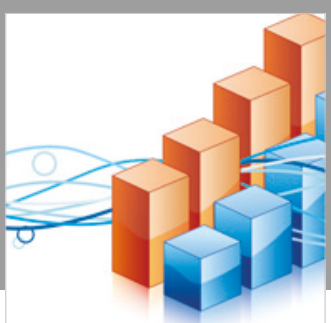

Advances in

Operations Research

\section{-n-m}
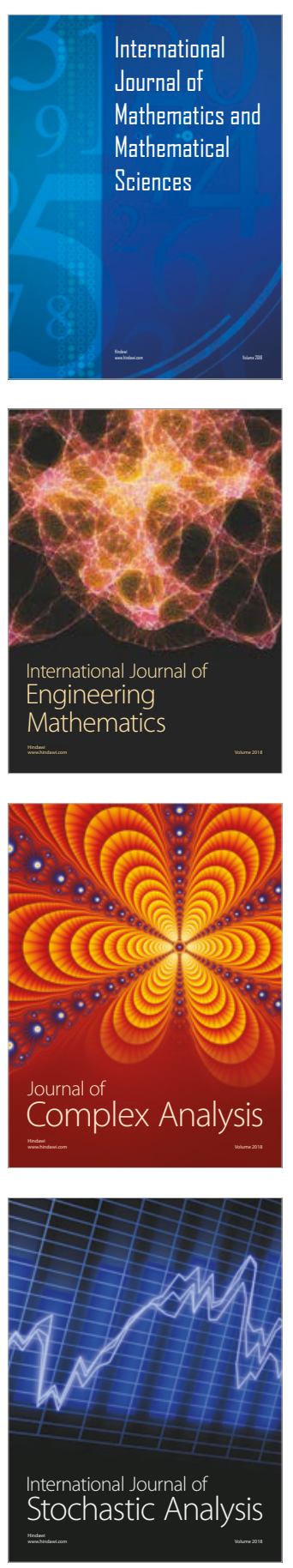
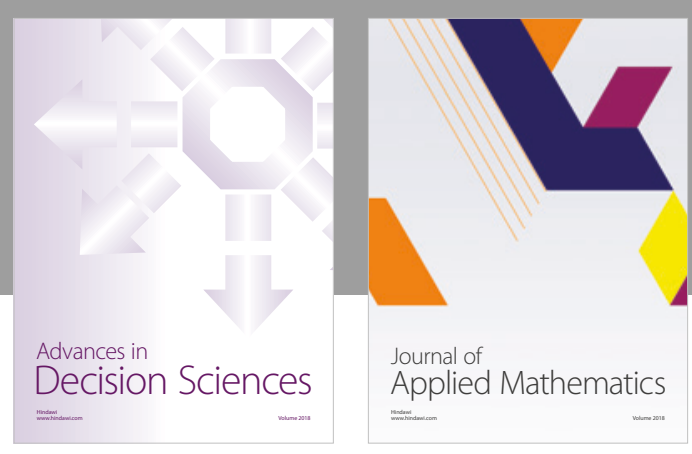

Journal of

Applied Mathematics
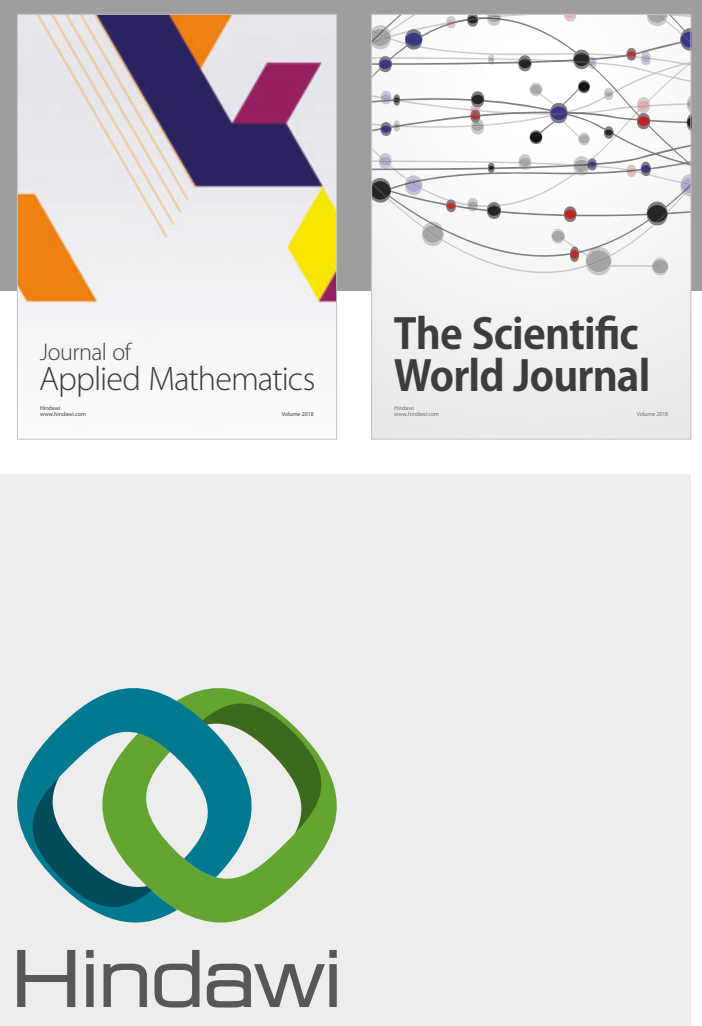

Submit your manuscripts at

www.hindawi.com

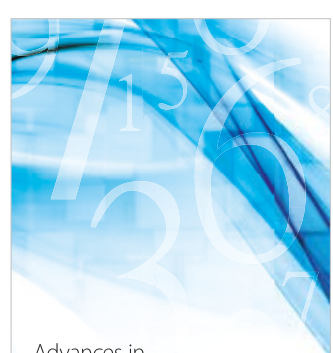

Advances in
Numerical Analysis
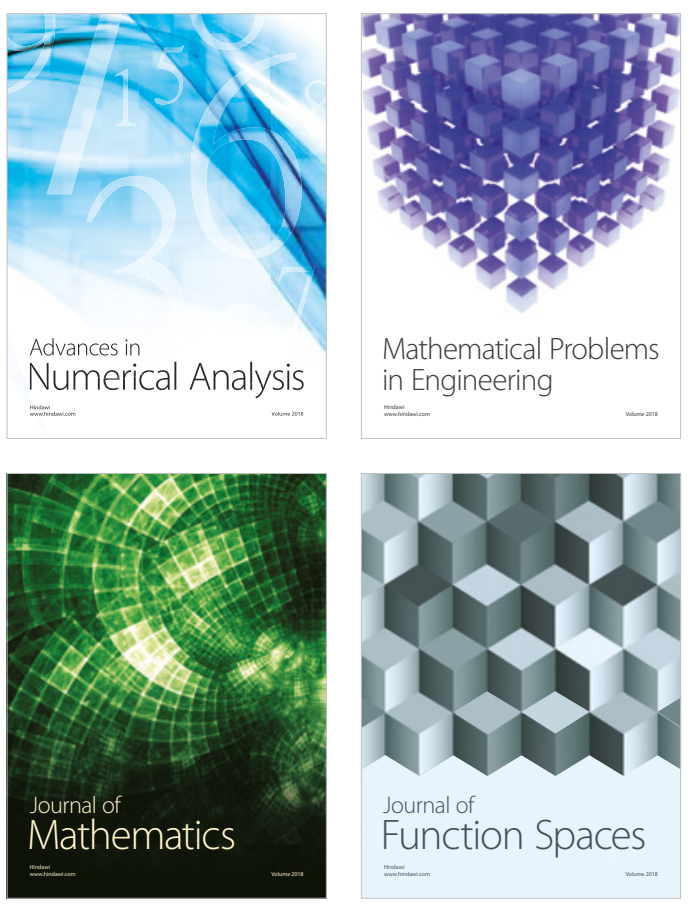

Mathematical Problems in Engineering

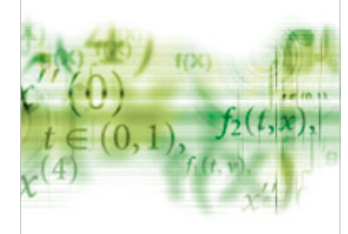

International Journal of

Differential Equations

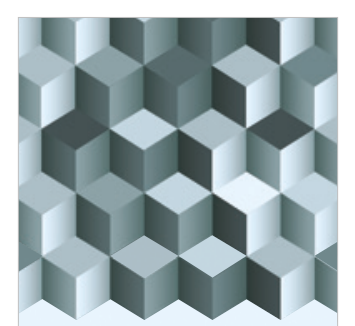

Journal of

Function Spaces

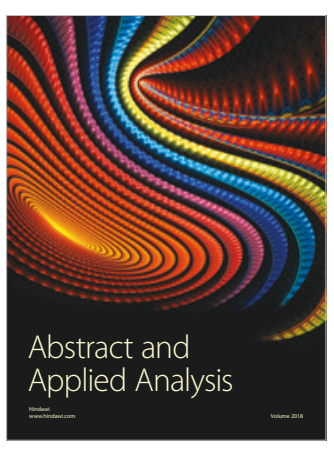

The Scientific

World Journal

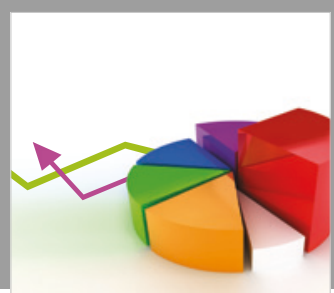

Journal of

Probability and Statistics
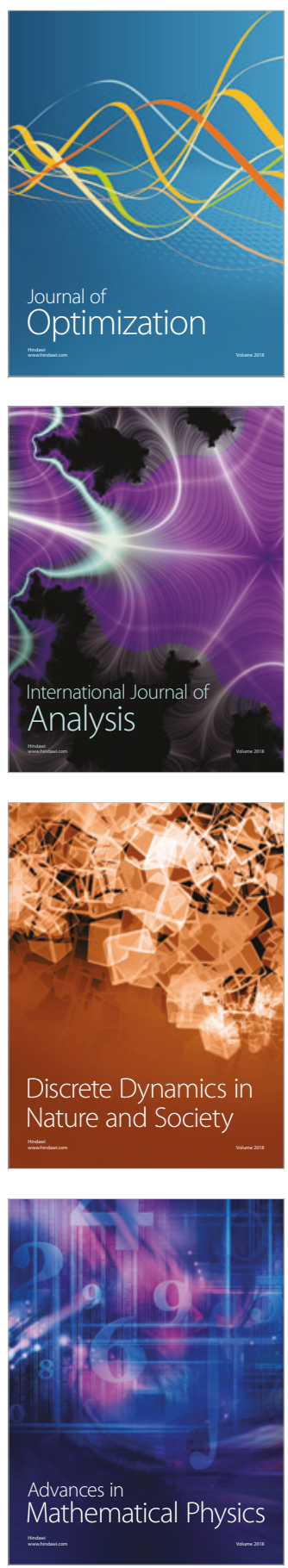